\title{
IDEAL STRUCTURE OF CROSSED PRODUCTS BY ENDOMORPHISMS VIA REVERSIBLE EXTENSIONS OF $C^{*}$-DYNAMICAL SYSTEMS
}

\author{
B. K. KWAŚNIEWSKI
}

\begin{abstract}
We consider an extendible endomorphism $\alpha$ of a $C^{*}$-algebra $A$. We associate to it a canonical $C^{*}$-dynamical system $(B, \beta)$ that extends $(A, \alpha)$ and is 'reversible' in the sense that the endomorphism $\beta$ admits a unique regular transfer operator $\beta_{*}$. The theory for $(B, \beta)$ is analogous to the theory of classic crossed products by automorphisms, and the key idea is to describe the counterparts of classic notions for $(B, \beta)$ in terms of the initial system $(A, \alpha)$.

We apply this idea to study the ideal structure of a non-unital version of the crossed product $C^{*}(A, \alpha, J)$ introduced recently by the author and A. V. Lebedev. This crossed product depends on the choice of an ideal $J$ in $(\operatorname{ker} \alpha)^{\perp}$, and if $J=(\operatorname{ker} \alpha)^{\perp}$ it is a modification of Stacey's crossed product that works well with non-injective $\alpha$ 's.

We provide descriptions of the lattices of ideals in $C^{*}(A, \alpha, J)$ consisting of gauge-invariant ideals and ideals generated by their intersection with $A$. We investigate conditions under which these lattices coincide with the set of all ideals in $C^{*}(A, \alpha, J)$. In particular, we obtain simplicity criteria that besides minimality of the action require either outerness of powers of $\alpha$ or pointwise quasinilpotence of $\alpha$.
\end{abstract}

\section{CONTENTS}

1. Introduction.

1.1. Preliminaries and notation

2. The category of $C^{*}$-dynamical systems

2.1. Reversible $C^{*}$-dynamical system

2.2. Extensions and covariant morphisms

3. Natural reversible extensions of $C^{*}$-dynamical systems and invariant ideals

3.1. The main construction

3.2. Invariant ideals and $J$-pairs

3.3. Invariant ideals in natural reversible extensions

4. Representations of $C^{*}$-dynamical systems. Crossed products

4.1. Covariant representations and morphisms to reversible systems

4.2. Crossed products

4.3. Gauge-invariant ideals

4.4. Simplicity

4.5. Topological freeness for reversible $C^{*}$-dynamical systems

4.6. Commutative $C^{*}$-dynamical systems

5. Appendix: $C^{*}(A, \alpha, J)$ viewed as a relative Cuntz-Pimsner algebra

References

2010 Mathematics Subject Classification. 46L05.

Key words and phrases. $C^{*}$-algebra, endomorphism, crossed product, ideal structure, simplicity, reversible extension.

This research was supported by NCN grant number DEC-2011/01/D/ST1/04112 and by a Marie CurieSklodowska Intra European Fellowship within the 7th European Community Framework Programme, project: OperaDynaDual. 


\section{INTRODUCTION.}

The theory of crossed products of $C^{*}$-algebras by endomorphisms was initiated by the ideas of Cuntz [12]. The original constructions were spatial, involved injective endomorphisms and one of their main aims was to produce new examples of simple $C^{*}$-algebras [43], [13, [45], [39], [40], [46]. A universal definition of a crossed product by an endomorphism was given by Stacey [47. When adapted to semigroup context [9, [1] it was investigated by many authors in connection with Toeplitz algebras of semigroups of isometries [2], 34, Bost-Connes Hecke algebras arising from number fields [35, 6], phase transitions [32] or short exact sequences and tensor products [36].

Despite of these achievements one has to note that the universal definition proposed by Stacey, even though it makes sense for an arbitrary endomorphism, is not correct when the underlying endomorphism is not injective. The reason is that Stacey's (multiplicity one) crossed product $A \times{ }_{\alpha}^{1} \mathbb{N}$ is isometric. This means, cf. [47, Definition 3.1], that the endomorphism $\alpha$ implementing the dynamics on the $C^{*}$-algebra $A$ is represented in $A \times{ }_{\alpha}^{1} \mathbb{N}$ via the relation

$$
\iota(\alpha(a))=u \iota(a) u^{*}, \quad a \in A,
$$

where $u$ is an isometry in $M\left(A \times_{\alpha}^{1} \mathbb{N}\right)$, and $\iota: A \rightarrow A \times_{\alpha}^{1} \mathbb{N}$ is the universal homomorphism. This readily implies that $\iota$ cannot be injective when $\alpha$ is not injective. Accordingly, the crossed product $A \times{ }_{\alpha}^{1} \mathbb{N}$ in fact does not depend on $(A, \alpha)$ but on the 'smaller' quotient system $\left(A / R, \alpha_{R}\right)$ where $R=\operatorname{ker} \iota$ and $\alpha_{R}(a+R)=\alpha(a)+R$ is a monomorphism of $A / R$ (cf. Remark4.11 below). In other words, speaking about non-injective endomorphisms in this context has only formal character.

In the case $A$ is unital, the authors of 31 proposed an alternative construction of a $C^{*}$ algebra generated by a faithful copy of $A$ and an operator $u$ satisfying (1), so that $u$ is an isometry exactly when $\alpha$ is a monomorphism. The point of departure in [31] is the remark that the relation (1) imply that

$$
u \text { is a (power) partial isometry and } \quad u^{*} u \iota(a)=\iota(a) u^{*} u, \quad a \in A \text {. }
$$

The universal $C^{*}$-algebra generated by objects satisfying (1), and hence automatically (2), was introduced and studied (in the semigroup case) in [37. However, this algebra should be viewed as a certain Toeplitz extension of the $C^{*}$-algebra we seek; in particular it is not a generalization of the classic crossed product. As argued in [31], in order to obtain a smaller algebra one should impose an additional relation. This relation can be phrased in terms of objects appearing in (2) via the formula

$$
J=\left\{a \in A: u^{*} u \iota(a)=\iota(a)\right\}
$$

where $J$ is an ideal in $A$. Stipulating that $\iota$ is faithful, one deduces that $J$ must be contained in the annihilator $(\operatorname{ker} \alpha)^{\perp}$ of the kernel of $\alpha$. For any ideal $J$ in $(\operatorname{ker} \alpha)^{\perp}$ the crossed product $C^{*}(A, \alpha, J)$ studied in [31] is a universal $C^{*}$-algebra with respect to relations (11), (31). The appropriate modification of Stacey's crossed product is the $C^{*}$-algebra $C^{*}(A, \alpha):=$ $C^{*}\left(A, \alpha,(\operatorname{ker} \alpha)^{\perp}\right)$ corresponding to $J=(\operatorname{ker} \alpha)^{\perp}$. Significantly, see [31, the $C^{*}$-algebras $C^{*}(A, \alpha, J)$ can be naturally modeled as relative Cuntz-Pimsner algebras [38. In this picture Stacey's crossed product corresponds to Pimsner's original construction [44] while $C^{*}(A, \alpha)$ corresponds to Katsura's [19] modification of Pimsner's $C^{*}$-algebras.

We also note that there are good reasons for studying $C^{*}(A, \alpha, J)$ with $J$ varying from $\{0\}$ to $(\operatorname{ker} \alpha)^{\perp}$ rather than focusing only on the unrelative crossed product $C^{*}(A, \alpha)$. For instance: 1) when $J=\{0\}$ we arrive at the partial isometric crossed product of [37];2) as we will see below, quotients of the unrelative crossed product by gauge-invariant ideals in general have the form $C^{*}(A, \alpha, J)$ where $\left.J \subsetneq(\operatorname{ker} \alpha)^{\perp} ; 3\right)$ compressions of weighted composition operators 
often generate $C^{*}$-algebras of type $C^{*}(A, \alpha, J)$ where the dependence on $J$ is essential, cf. [24, Theorem. 5.6].

In the present paper, motivated by applications to non-unital $C^{*}$-algebras, cf. for instance [18, [36], we extend the definition of $C^{*}(A, \alpha, J)$. In our setting it applies to $C^{*}$-dynamical systems $(A, \alpha)$ consisting of a (possibly non-unital) $C^{*}$-algebra $A$ and an endomorphism $\alpha$ : $A \rightarrow A$ that extends to the strictly continuous endomorphism $\bar{\alpha}: M(A) \rightarrow M(A)$, cf. [1]. We call $(A, \alpha)$ a reversible $C^{*}$-dynamical system if $\alpha$ shifts $(\operatorname{ker} \alpha)^{\perp}$ onto a corner in $A$ and ker $\alpha$ is a complemented ideal in $A$. Then there exists a unique transfer operator $\alpha_{*}$ for $\alpha$ [15], [11], such that $\alpha_{*} \circ \alpha$ is a conditional expectation on $\alpha(A)$, cf. [23], [28]. For such systems the $C^{*}$-algebra $C^{*}(A, \alpha)$ coincides with Exel's crossed product $A \rtimes_{\alpha, \alpha_{*}} \mathbb{N}$, introduced in [15] and adapted to non-unital case in [11], see also [28]. The structure of $C^{*}(A, \alpha)=A \rtimes_{\alpha, \alpha_{*}} \mathbb{N}$ is relatively similar to that of the classic crossed product by an automorphism, see [3], [23, Remark 1.10], [27]. We note that in general the theories of Exel's crossed products and crossed products considered in this paper are different. As explained in [28] they can be naturally unified in the realm of crossed products by completely positive maps.

Our strategy to investigate $C^{*}(A, \alpha, J)$ is similar, but yet slightly different, to the very popular method of dilations and corners, see [33] as well as [12, 43], [13, [47, 45]. The latter method works only for injective endomorphisms and allows one to regard crossed products by endomorphisms as (full) corners in crossed products by automorphisms. Therefore it gives a realization of the crossed product only up to Morita equivalence. This might be too insensitive tool when studying more subtle problems such as, for instance, spectra of elements of the algebras under consideration.

In our approach we start with an arbitrary $C^{*}$-dynamical system $(A, \alpha)$ and an ideal $J$ in $(\operatorname{ker} \alpha)^{\perp}$. We construct a canonical reversible $C^{*}$-dynamical system $(B, \beta)$ that extends $(A, \alpha)$. We call $(B, \beta)$ a natural reversible $J$-extension of $(A, \alpha)$. It is defined as a direct limit in the category of $C^{*}$-dynamical systems, and identified as a universal object in the sense to be specified below. Examples of such objects in the commutative case were studied in [30, [24, and when $A$ is unital were constructed in [29]. In particular, it follows that we have a natural isomorphism

$$
C^{*}(A, \alpha, J) \cong C^{*}(B, \beta) .
$$

Our main goal is to use the above isomorphism to get a description of the ideal structure of $C^{*}(A, \alpha, J)$. The tactics is as follows. Firstly, we develop or extend to non-unital case the tools that apply to $C^{*}(B, \beta)$, cf. [27], [3]. Secondly, by an analysis of the relationship between $(A, \alpha)$ and $(B, \beta)$ we derive results for general crossed products $C^{*}(A, \alpha, J)$. The general relationship, established in this way, between the lattices of ideals of various type is presented by the diagram on Figure 1, here the arrow $A \Longrightarrow B$ indicates that there is an order retraction 1 from the lattice $A$ onto the lattice $B$, and $A \Longleftrightarrow B$ means that $A$ and $B$ are order isomorphic (precise descriptions will be given below).

For reversible $C^{*}$-dynamical systems the theory looks quite similar to the classic theory for automorphic actions. For instance, adopting parts of [27] to non-unital case, we identify a condition on $(B, \beta)$ under which all ideals in $C^{*}(A, \alpha, J)$ are gauge-invariant (see Corollary 4.24 below). One of the difficult problems is to phrase such conditions in terms of $(A, \alpha)$ and $J$, which we do in full generality in the commutative case (Proposition 4.36). Moreover, as it can be well seen from our analysis, an essential difference comparing to the classic theory arise for systems $(A, \alpha)$ where the kernel ker $\alpha$ is not a complemented ideal in $A$. For such systems, even in the unrelative crossed product $C^{*}(A, \alpha)$, not all gauge-invariant ideals are generated by their intersection with $A$. In order to describe such ideals in general (Theorem 4.15) we need

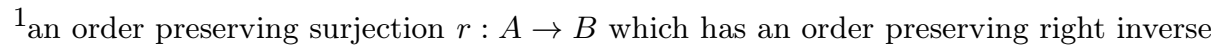




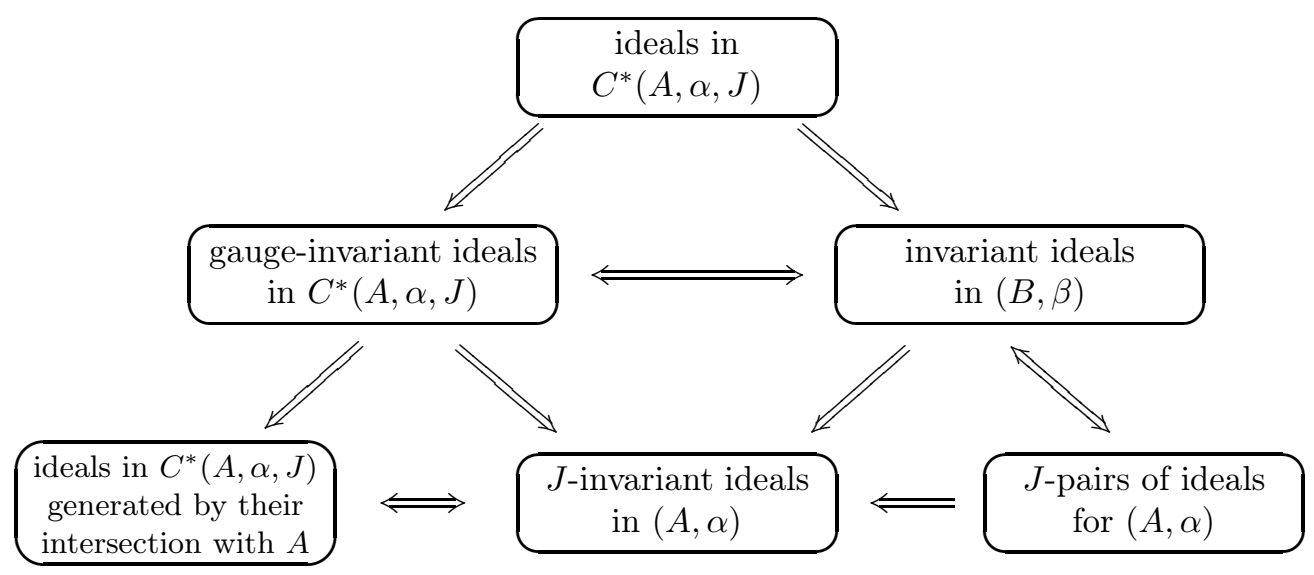

Figure 1. Relationships between the lattices of ideals

a pair of ideals in $A$. Similar facts were first observed in the context of graph $C^{*}$-algebras [7] and for general relative Cuntz-Pimsner are described in [21].

Another new phenomenon occurs in the study of simplicity of $C^{*}(A, \alpha, J)$ (Theorem 4.18). We show that $C^{*}(A, \alpha, J)$ is not simple unless it is the unrelative crossed product $C^{*}(A, \alpha)$ and $(A, \alpha)$ is minimal. The minimality condition we use (Definition 4.17) is slightly more subtle then the corresponding condition used in the unital case, see [43, [39], [40, [46]. Moreover, the simplicity of $C^{*}(A, \alpha)$ implies the following dynamical dichotomy: either

1) $\alpha$ is pointwise quasinilpotent, or

2) $\alpha$ is a monomorphism and no power $\alpha^{n}, n>0$, is inner.

Conversely, for a minimal $(A, \alpha)$ we show that 1$)$ is sufficient for simplicity of $C^{*}(A, \alpha)$, and if $A$ is unital we infer from [46. Theorem 4.1] that 2) implies $C^{*}(A, \alpha)$ is simple. We also examine condition 2) in the non-unital case, for reversible systems and arbitrary systems on commutative algebras (see respectively Corollary 4.25 and Theorem 4.37). We remark that condition 2) is related to the classic theorem of Kishimoto [22] while 1) can not occur for non-injective $\alpha$.

It is fair to say that we could analyze $C^{*}(A, \alpha, J)$ as a relative Cuntz-Pimsner algebra (or even as a $C^{*}$-algebra of an ideal in a right tensor $C^{*}$-precategory, cf. [25, Example 2.20]). Such analysis would heavily rely on the results of [21]; we briefly explain it in the appendix section. However, our direct approach has several advantages. It makes the content accessible for the broader audience and allows the presentation to be self-contained. Hopefully, more direct arguments shed more light on the investigated problems and open the door to further future generalizations, for instance, in the purely algebraic setting, cf. [5]. Last but not least, we think that the method of using reversible extensions developed in the paper has a potential. For example, we plan to use it in forthcoming papers to tackle endomorphisms of $C_{0}(X)$-algebras and to analyze spectra of the related weighted composition operators, cf. [4].

The content of the paper is essentially divided into two parts.

Sections 2 and 3 are 'space free' - all considerations are performed in a category of $C^{*}$ dynamical systems $C^{*}$-dyna. In section 2 we discus reversible $C^{*}$-dynamical systems which are special objects in $C^{*}$-dyna. We also introduce and study the notion of $J$-covariance for morphisms in $C^{*}$-dyna. In section 3 we construct the natural reversible $J$-extension $(B, \beta)$ of a $C^{*}$-dynamical system $(A, \alpha)$ as a certain direct limit in $C^{*}$-dyna, and characterize it as a universal object (Theorem 3.1). Invariant ideals and $J$-pairs for $(A, \alpha)$ are introduced in subsection 3.2. The important tool is Theorem 3.12 where we describe invariant ideals in $(B, \beta)$, and the corresponding quotient subsystems, in terms of $J$-pairs for $(A, \alpha)$. 
In Section 4 we investigate representation theory for $C^{*}$-dynamical systems. In subsection 4.1 we generalize the notion of a $J$-covariant representation of $(A, \alpha)$, 31, Definition 1.7], and show that such representations can be treated as $J$-covariant morphisms from $(A, \alpha)$ to reversible $C^{*}$-dynamical systems. This allows us to apply the previously elaborated tools to study the crossed product $C^{*}(A, \alpha, J)$, which we define in subsection 4.2. In particular, we describe the lattice of its gauge-invariant ideals in Theorem 4.15 and establish the simplicity criteria in Theorem 4.18. In subsection 4.5 we exploit the notion of topological freeness defined for reversible $C^{*}$-dynamical systems. Finally, we illustrate the theory with a discussion of the commutative case, see subsection 4.6.

We close the paper with an appendix indicating how to analyze $C^{*}(A, \alpha, J)$ using the general theory of relative Cuntz-Pimsner algebras. We focus here mainly on the ideal structure, but this also leads to criteria for other properties of $C^{*}(A, \alpha, J)$ such as nuclearity or exactness.

1.1. Preliminaries and notation. All ideals in $C^{*}$-algebras are assumed to be closed and two sided. We denote by $I^{\perp}=\{a \in A: a I=\{0\}\}$ the annihilator of an ideal $I$ in a $C^{*}$-algebra $A$ (it is the largest ideal in $A$ with the property that $I^{\perp} \cap I=\{0\}$ ). An ideal $I$ in $A$ is called essential if $I^{\perp}=\{0\}$. A multiplier algebra $M(A)$ of $A$ can be characterized as a maximal unital $C^{*}$-algebra containing $A$ as an essential ideal. We will denote the unit in $M(A)$ simply by 1 . Occasionally we will use the same symbol for units in different algebras but this should not cause confusion. One of the models for $M(A)$ is where the elements of $M(A)$ are viewed as functions on $A$ possessing (necessarily unique) adjoints:

$$
M(A)=\left\{m: A \rightarrow A: \exists_{m^{*}: A \rightarrow A}(m a)^{*} b=a^{*}\left(m^{*} b\right) \text { for all } a, b \in A\right\} .
$$

Another approach is to view $M(A)$ as a completion of $A$ in the strict topology. A net $m_{\lambda}$ in $M(A)$ converges strictly to $m \in M(A)$ if and only if $m_{\lambda} a \rightarrow m a$ and $m_{\lambda}^{*} a \rightarrow m^{*} a$ in $A$, for any $a \in A$. Since we will need notation only for closures in the strict topology we adopt the convention that for any subset $I$ of $A$

$$
\bar{I} \text { stands for the strict closure of } I \subseteq A \text { in } M(A) \text {. }
$$

By homomorphisms, epimorphisms, etc. between $C^{*}$-algebras we always mean $*$-preserving maps. A continuous mapping $T: A \rightarrow B$ between $C^{*}$-algebras $A$ and $B$ is said to be extendible, cf. [1], if it extends to a (necessarily unique) strictly continuous mapping $\bar{T}: M(A) \rightarrow M(B)$. A homomorphism $T: A \rightarrow B$ is extendible if and only if for some (and hence any) approximate unit $\left\{\mu_{\lambda}\right\}$ in $A$ the net $\left\{T\left(\mu_{\lambda}\right)\right\}$ converges strictly in $M(B)$. Then $\bar{T}$ is determined by the formula $\bar{T}(m) b=\lim T\left(m \mu_{\lambda}\right) b, m \in M(A), b \in B$. If the net $\left\{T\left(\mu_{\lambda}\right)\right\}$ converges strictly to the unit in $M(B)$ homomorphism $T$ is called non-degenerate.

The following relations between strict closures and strictly continuous extensions will be of constant use in the paper.

Lemma 1.1. Let $I$ be an ideal in $A$ and $\bar{I}$ its strict closure in $M(A)$. We have

$$
\bar{I}=\{b \in M(A): b A \subseteq I\} \quad \text { and } \quad \bar{I}^{\perp}=\overline{I^{\perp}}
$$

where $\bar{I}^{\perp}$ is the annihilator of $\bar{I}$ in $M(A)$ and $\overline{I^{\perp}}$ is the strict closure of the annihilator of $I$ in $A$. If $T: A \rightarrow B$ is an extendible homomorphism, then

$$
\operatorname{ker} \bar{T}=\overline{\operatorname{ker} T}, \quad(\operatorname{ker} \bar{T})^{\perp}=\overline{(\operatorname{ker} T)^{\perp}} .
$$

Proof. Clearly $\{b \in M(A): b A \subseteq I\}$ is strictly closed and contains $I$, whence $\bar{I} \subseteq\{b \in M(A)$ : $b A \subseteq I\}$. Moreover, if $b \in M(A)$ is such that $b A \subseteq I$ and $\left\{\mu_{\lambda}\right\}$ is an approximate unit in $A$, then $\left\{b \mu_{\lambda}\right\} \subseteq I$ is a net converging strictly to $b$. Therefore $\bar{I}=\{b \in M(A): b A \subseteq I\}$. Now let 
$b \in M(A)$. Using what we have just proved we get

$$
\begin{aligned}
b \in \bar{I}^{\perp} & \Longleftrightarrow b \bar{I}=\{0\} \Longleftrightarrow b \bar{I} A=\{0\} \Longleftrightarrow b I=\{0\} \Longleftrightarrow b A I=\{0\} \Longleftrightarrow b A \subseteq I^{\perp} \\
& \Longleftrightarrow b \in \overline{I^{\perp}} .
\end{aligned}
$$

This shows the first part of the assertion. For the second part note that we have $\overline{\operatorname{ker} T} \subseteq \operatorname{ker} \bar{T}$, and the reverse inclusion follows because

$$
b \in \operatorname{ker} \bar{T} \Longrightarrow T(b A)=\bar{T}(b) T(A)=0 \Longrightarrow b A \subseteq \operatorname{ker} T \Longrightarrow b \in \overline{\operatorname{ker} T} .
$$

Now we get $(\operatorname{ker} \bar{T})^{\perp}=(\overline{\operatorname{ker} T})^{\perp}=\overline{(\operatorname{ker} T)^{\perp}}$.

If $I$ is an ideal in $A$ we denote by $q_{I}: A \rightarrow A / I$ the quotient map, and occasionally when the context is clear we suppress the subscript $I$ and write simply $q$. We extend the standard notation concerning the sum of ideals in $C^{*}$-algebras, and if $D_{0}, D_{2}, . ., D_{n}, \ldots$ are linear subspaces in a $C^{*}$-algebra we put

$$
\sum_{k=0}^{n} D_{k}:=\left\{\sum_{k=0}^{n} d_{k}: d_{k} \in D_{k}\right\} \quad \text { and } \quad \sum_{k=0}^{\infty} D_{k}:=\overline{\operatorname{span}}\left\{d_{k}: d_{k} \in D_{k}, k \in \mathbb{N}\right\} .
$$

Hence $\sum_{k=0}^{\infty} D_{k}$ is the closure of the increasing sum of the spaces $\sum_{k=0}^{n} D_{k}, n \in \mathbb{N}$. Similarly, we put $C D:=\{c d: c \in C, b \in D\}$ whenever an action of $C$ on $D$ makes sense. By CohenHewitt factorization theorem a homomorphism $T: A \rightarrow B$ between $C^{*}$-algebras $A$ and $B$ is non-degenerate if and only if $T(A) B=B$.

\section{The CATEGory of $C^{*}$-DYNAMiCAL SYSTEMS}

We start by introducing the category of $C^{*}$-dynamical systems which we will work with.

Definition 2.1. A $C^{*}$-dynamical system is a pair $(A, \alpha)$ where $A$ is a $C^{*}$-algebra and $\alpha: A \rightarrow A$ is an extendible endomorphism. A morphism from a $C^{*}$-dynamical system $(A, \alpha)$ to a $C^{*}$ dynamical system $(B, \beta)$ is a non-degenerate homomorphism $T: A \rightarrow B$ such that $T \circ \alpha=\beta \circ T$.

We will signalize this by writing $(A, \alpha) \stackrel{T}{\rightarrow}(B, \beta)$. The arising category will be denoted by $C^{*}$-dyna.

Passage to strictly continuous extensions yields a functor from $C^{*}$-dyna onto its full subcategory whose objects are endomorphisms of unital $C^{*}$-algebras.

Lemma 2.2. If $(A, \alpha) \stackrel{T}{\rightarrow}(B, \beta)$ then $(M(A), \bar{\alpha}) \stackrel{\bar{T}}{\rightarrow}(M(B), \bar{\beta})$.

Proof. Clear by strict density of $A$ in $M(A)$ and strict continuity of $\bar{\alpha}, \bar{\beta}$ and $\bar{T}$.

2.1. Reversible $C^{*}$-dynamical system. We distinguish certain objects in $C^{*}$-dyna which will view as reversible systems. In the commutative case they correspond to partial homeomorphisms, see [30, 24, and subsection 4.6 below. In noncommutative setting the role of an inverse to an endomorphism plays a complete transfer operator.

Transfer operators [15] and complete transfer operators [3] were originally introduced for unital $C^{*}$-algebras. Nevertheless, they can be easily adapted to our context. Let $(A, \alpha)$ be a $C^{*}$-dynamical system. A transfer operator for $(A, \alpha)$ is a positive linear map $L: A \rightarrow A$ such that

$$
L(\alpha(a) b)=a L(b), \quad \text { for all } a, b \in A .
$$

The transfer operator $L$ is a complete transfer operator if it satisfies

$$
\alpha(L(a))=\bar{\alpha}(1) a \bar{\alpha}(1), \quad \text { for all } a \in A .
$$

The following statement could be deduced from the results of [28], cf. also [23]. For the sake of completeness we give an independent proof. 
Proposition 2.3. A $C^{*}$-dynamical system $(A, \alpha)$ admits a complete transfer operator if and only if the range $\alpha(A)$ is a hereditary subalgebra of $A$ (hence a corner) and the kernel ker $\alpha$ is a complemented ideal in $A$.

Moreover, if it exists, the complete transfer operator for $(A, \alpha)$ is unique and is given by the formula

$$
\alpha_{*}(a)=\alpha^{-1}(\bar{\alpha}(1) a \bar{\alpha}(1)), \quad a \in A,
$$

where $\alpha^{-1}$ is the inverse to the isomorphism $\alpha:(\operatorname{ker} \alpha)^{\perp} \rightarrow \alpha(A)=\bar{\alpha}(1) A \bar{\alpha}(1)$.

Proof. Suppose that $\alpha_{*}$ is a complete transfer operator for $(A, \alpha)$. It follows from (5) that $\alpha(A)=\bar{\alpha}(1) A \bar{\alpha}(1)$ and $\alpha \circ \alpha_{*} \circ \alpha=\alpha$. Hence $\alpha(A)$ is a hereditary subalgebra of $A$ and the map $p:=\alpha_{*} \circ \alpha$ is an idempotent acting on $A$. Relations $\alpha=\alpha \circ p$ and $p=\alpha_{*} \circ \alpha$ imply that $\operatorname{ker} p=\operatorname{ker} \alpha$. Moreover, (4) implies that $\alpha_{*}(A) \subseteq(\operatorname{ker} \alpha)^{\perp}$ and hence $p(A) \subseteq(\operatorname{ker} \alpha)^{\perp}$. Since $p$ is an idempotent this shows that $p(A)=(\operatorname{ker} \alpha)^{\perp}$. As a consequence we also get $\alpha_{*}(A)=(\operatorname{ker} \alpha)^{\perp}$. To prove that $\operatorname{ker} \alpha$ is a complemented ideal it suffices to show that $p$ is multiplicative. Since $\alpha$ is injective on $p(A)=(\operatorname{ker} \alpha)^{\perp}$, this follows from the calculation

$$
\alpha(p(a \cdot b))=\alpha(a \cdot b)=\alpha(a) \alpha(b)=\alpha(p(a)) \alpha(p(b))=\alpha(p(a) \cdot p(b))
$$

where $a, b \in A$. In particular, $\alpha:(\operatorname{ker} \alpha)^{\perp} \rightarrow \bar{\alpha}(1) A \bar{\alpha}(1)$ is an isomorphism. Taking into account (5) and $\alpha_{*}(A)=(\operatorname{ker} \alpha)^{\perp}$ we get (6) .

Conversely, if $\alpha(A)$ is hereditary in $A$ it is automatically the corner $\bar{\alpha}(1) A \bar{\alpha}(1)$. If additionally the ideal ker $\alpha$ is complemented then $\alpha:(\operatorname{ker} \alpha)^{\perp} \rightarrow \bar{\alpha}(1) A \bar{\alpha}(1)$ is an isomorphism. In this case one easily checks that (6) defines a complete transfer operator.

Definition 2.4. If a $C^{*}$-dynamical system $(A, \alpha)$ possess the (necessarily unique) complete transfer operator we denote it by $\alpha_{*}$ and we call $(A, \alpha)$ a reversible $C^{*}$-dynamical system.

Remark 2.5. It readily follows from (6) that the complete transfer operator $\alpha_{*}$ is a generalized inverse to $\alpha$, that is we have $\alpha=\alpha \circ \alpha_{*} \circ \alpha$ and $\alpha_{*}=\alpha_{*} \circ \alpha \circ \alpha_{*}$. Moreover, one can show, cf. [28], that if the complete transfer operator exists it is the unique transfer operator satisfying $\alpha=\alpha \circ \alpha_{*} \circ \alpha$.

A complete transfer operator always admits a strictly continuous extension and in particular is a transfer operator in the sense of [11], cf. [28].

Proposition 2.6. $A C^{*}$-dynamical system $(A, \alpha)$ is reversible if and only if the extended system $(M(A), \bar{\alpha})$ is reversible.

If $(A, \alpha)$ is reversible, the complete transfer operator $\bar{\alpha}_{*}$ for $(M(A), \bar{\alpha})$ is a strict continuous extension of $\alpha_{*}$, and $\left\{\bar{\alpha}_{*}^{n}(1)\right\}_{n \in \mathbb{N}}$ is a decreasing sequence of central projections in $M(A)$ with $\bar{\alpha}_{*}^{n}(1) A=\operatorname{ker}\left(\alpha^{n}\right)^{\perp}$.

Proof. By Lemma 1.1, $\operatorname{ker} \bar{\alpha}=\overline{\operatorname{ker} \alpha}$. Thus $\operatorname{ker} \alpha$ is complemented in $A$ if and only if $\operatorname{ker} \bar{\alpha}$ is complemented in $M(A)$. Now, if $(M(A), \bar{\alpha})$ is reversible then $\alpha(A)=\bar{\alpha}(A)=\bar{\alpha}\left(\bar{\alpha}_{*}(A)\right)=$ $\bar{\alpha}(1) A \bar{\alpha}(1)$ is hereditary in $A$, and hence $(A, \alpha)$ is reversible by Proposition 2.3 . Conversely, if $(A, \alpha)$ is reversible then for any net $\left\{a_{\lambda}\right\} \subseteq A$ strictly convergent to $a \in M(A)$ the net $\left\{\bar{\alpha}(1) a_{\lambda} \bar{\alpha}(1)\right\}=\left\{\alpha\left(\alpha_{*}\left(a_{\lambda}\right)\right)\right\} \subseteq \bar{\alpha}(M(A))$ is convergent to $\bar{\alpha}(1) a \bar{\alpha}(1)$. It follows that $\bar{\alpha}(M(A))=$ $\bar{\alpha}(1) M(A) \bar{\alpha}(1)$ is hereditary in $M(A)$ and hence $(M(A), \bar{\alpha})$ is reversible by Proposition 2.3 .

Suppose now that the systems $(A, \alpha)$ and $(M(A), \bar{\alpha})$ are reversible. Then $\bar{\alpha}_{*}$ is a strictly continuous extension of $\alpha_{*}$ because it is given by (6) where $\alpha^{-1}$ is replaced by the the inverse to the strictly continuous isomorphism $\bar{\alpha}: \overline{(\operatorname{ker} \alpha)^{\perp}}=(\operatorname{ker} \bar{\alpha})^{\perp} \rightarrow \overline{\alpha(A)}=\bar{\alpha}(1) M(A) \bar{\alpha}(1)$. Furthermore, for each $n \in \mathbb{N}, \alpha_{*}^{n}$ is the complete transfer operator for $\left(A, \alpha^{n}\right)$. In particular, $\bar{\alpha}_{*}^{n}(1)$ is a projection onto $\operatorname{ker}\left(\bar{\alpha}^{n}\right)^{\perp}$. 


\subsection{Extensions and covariant morphisms.}

Definition 2.7. If $T$ is an injective morphism $(A, \alpha) \stackrel{T}{\rightarrow}(B, \beta)$ we call it an embedding of $(A, \alpha)$ into $(B, \beta)$, and say that $(B, \beta)$ is an extension of $(A, \alpha)$. If $T$ is an isomorphism then $(A, \alpha)$ and $(B, \beta)$ are equivalent (in the category $C^{*}$-dyna).

Note that if $T$ is an embedding of $(A, \alpha)$ to $(B, \beta)$ then

$$
\operatorname{ker} \alpha=T^{-1}(\operatorname{ker} \beta), \quad(\operatorname{ker} \alpha)^{\perp} \supseteq T^{-1}\left((\operatorname{ker} \beta)^{\perp}\right) .
$$

These relations could be interpreted as follows: the extended endomorphism $\beta$ may enlarge the kernel of $\alpha$ but only outside $A$, and if it does, it may shrink the annihilator of the kernel inside $A$. Thus the ideal $J=T^{-1}\left((\operatorname{ker} \beta)^{\perp}\right)$ is a parameter that measures how far can $\operatorname{ker} \beta$ from ker $\alpha$ go. We have $\{0\} \subseteq J \subseteq(\operatorname{ker} \alpha)^{\perp}$ and when $J=(\operatorname{ker} \alpha)^{\perp}$ the relationship between the kernels is the strongest. This leads us to the following definition.

Definition 2.8. Let $J$ be an ideal in $(\operatorname{ker} \alpha)^{\perp}$. We will say that $(B, \beta)$ is a $J$-covariant extension of $(A, \alpha)$, or briefly a $J$-extension of $(A, \alpha)$, if there exits an embedding $(A, \alpha) \stackrel{T}{\rightarrow}(B, \beta)$ such that

$$
J=T^{-1}\left((\operatorname{ker} \beta)^{\perp}\right) .
$$

In this event $T$ will be called a $J$-covariant embedding, or briefly $J$-embedding, of $(A, \alpha)$ into $(B, \beta)$. If $J=(\operatorname{ker} \alpha)^{\perp}$ instead of $J$-covariant extension and $J$-covariant embedding we will use terms covariant extension and covariant embedding.

It is useful to extend the above notion of covariance to (not necessarily injective) morphisms $(A, \alpha) \stackrel{T}{\rightarrow}(B, \beta)$. But, in general, since $\operatorname{ker} T \subseteq T^{-1}\left((\operatorname{ker} \beta)^{\perp}\right)$ one can not expect to have equality (77) for an ideal $J$ in $(\operatorname{ker} \alpha)^{\perp}$. One of possible ways to circumvent this problem is to replace (7) by an inclusion.

Definition 2.9. Let $J$ be an ideal in $(\operatorname{ker} \alpha)^{\perp}$. We say that a morphism $(A, \alpha) \stackrel{T}{\rightarrow}(B, \beta)$ is covariant on $J$ if

$$
J \subseteq T^{-1}\left((\operatorname{ker} \beta)^{\perp}\right)
$$

We say $T$ is covariant if it is covariant on $(\operatorname{ker} \alpha)^{\perp}$.

Remark 2.10. An embedding $T$ is $J$-covariant if and only if $J$ is the largest ideal on which $T$ is covariant as a morphism. In particular, $T$ is a covariant embedding if and only if $T$ is an injective covariant morphism.

We show now certain properties of extensions and morphisms we will use latter. They already indicate a special role played by covariant morphisms.

Lemma 2.11. If $(A, \alpha) \stackrel{T}{\rightarrow}(B, \beta)$ is morphism, then

$$
\overline{T^{-1}\left((\operatorname{ker} \beta)^{\perp}\right)}=\bar{T}^{-1}\left((\operatorname{ker} \bar{\beta})^{\perp}\right),
$$

recall that the bar on the left-hand side denotes the strict closure. In particular,

i) $(A, \alpha) \stackrel{T}{\rightarrow}(B, \beta)$ is covariant on $J \subseteq(\operatorname{ker} \alpha)^{\perp}$ iff $(M(A), \bar{\alpha}) \stackrel{\bar{T}}{\rightarrow}(M(B), \bar{\beta})$ is covariant on $\bar{J} \subseteq(\operatorname{ker} \bar{\alpha})^{\perp}$,

ii) $(A, \alpha) \stackrel{T}{\rightarrow}(B, \beta)$ is $J$-covariant embedding iff $(M(A), \bar{\alpha}) \stackrel{\bar{T}}{\rightarrow}(M(B), \bar{\beta})$ is $\bar{J}$-covariant embedding,

iii) $(A, \alpha) \stackrel{T}{\rightarrow}(B, \beta)$ is a covariant morphism iff $(M(A), \bar{\alpha}) \stackrel{\bar{T}}{\rightarrow}(M(B), \bar{\beta})$ is a covariant morphism. 
Proof. By Lemma 1.1 we have $(\operatorname{ker} \bar{\beta})^{\perp}=\overline{(\operatorname{ker} \beta)^{\perp}}$. Hence

$$
T^{-1}\left((\operatorname{ker} \beta)^{\perp}\right) \subseteq \bar{T}^{-1}\left(\left(\overline{\operatorname{ker} \beta)^{\perp}}\right) \Longrightarrow \overline{T^{-1}\left((\operatorname{ker} \beta)^{\perp}\right)} \subseteq \bar{T}^{-1}\left((\operatorname{ker} \bar{\beta})^{\perp}\right) .\right.
$$

For the reverse inclusion note that for any $b \in \bar{T}^{-1}\left((\operatorname{ker} \bar{\beta})^{\perp}\right)$ we have

$$
T(b A)=\bar{T}(b) T(A) \subseteq(\operatorname{ker} \bar{\beta})^{\perp} \cap B=\overline{(\operatorname{ker} \beta)^{\perp}} \cap B=(\operatorname{ker} \beta)^{\perp} .
$$

Hence $b A \subseteq T^{-1}\left((\operatorname{ker} \beta)^{\perp}\right)$ and therefore $b \in \overline{T^{-1}\left((\operatorname{ker} \beta)^{\perp}\right)}$ by Lemma 1.1. This proves (8) . The second part of the assertion is now straightforward.

Lemma 2.12. Let $(A, \alpha) \stackrel{T}{\rightarrow}(B, \beta)$ and suppose that $\operatorname{ker} \alpha$ and $\operatorname{ker} \beta$ are complemented ideals in $A$ and $B$, respectively. The following conditions are equivalent:

i) $T$ is covariant,

ii) $\bar{T}\left(1_{(\operatorname{ker} \alpha)^{\perp}}\right) \leq 1_{(\operatorname{ker} \beta)^{\perp}}$,

iii) $\bar{T}\left(1_{(\operatorname{ker} \alpha)^{\perp}}\right)=1_{(\operatorname{ker} \beta)^{\perp}}$,

where $1_{(\operatorname{ker} \alpha)^{\perp}} \in M(A)$ and $1_{(\operatorname{ker} \beta)^{\perp}} \in M(B)$ denote the projections onto $(\operatorname{ker} \alpha)^{\perp}$ and $(\operatorname{ker} \beta)^{\perp}$, respectively.

Proof. By passing to extended systems, see Lemma 2.11 iii), we may assume that both $A$ and $B$ are unital. Then $T$ is unital and $1_{(\operatorname{ker} \alpha)^{\perp}}$ and $1_{(\operatorname{ker} \beta)^{\perp}}$ are units in $(\operatorname{ker} \alpha)^{\perp}$ and $(\operatorname{ker} \beta)^{\perp}$, respectively. Now, one readily sees that the inclusion $(\operatorname{ker} \alpha)^{\perp} \subseteq T^{-1}\left((\operatorname{ker} \beta)^{\perp}\right)$ is equivalent to the inequality $T\left(1_{(\operatorname{ker} \alpha)^{\perp}}\right) \leq 1_{(\operatorname{ker} \beta)^{\perp}}$. Hence i) is equivalent to ii). Moreover, $T \circ \alpha=\beta \circ T$ implies that $T\left(1_{\operatorname{ker} \alpha}\right) \leq 1_{\operatorname{ker} \beta}$ where $1_{\operatorname{ker} \alpha}$ and $1_{\operatorname{ker} \beta}$ are units in $\operatorname{ker} \alpha$ and ker $\beta$, respectively. Thus, as $T$ is unital, the inequality $T\left(1_{(\operatorname{ker} \alpha)^{\perp}}\right) \geq 1_{(\operatorname{ker} \beta)^{\perp}}$ is always satisfied. Hence ii) is equivalent to iii).

In connection with the following statements recall, see Proposition 2.6, that if $\alpha_{*}$ is a complete transfer operator for $(A, \alpha)$, then $\bar{\alpha}_{*}(1)$ is the unit in $(\operatorname{ker} \bar{\alpha})^{\perp}=\overline{(\operatorname{ker} \alpha)^{\perp}}$.

Proposition 2.13. Suppose $(A, \alpha)$ and $(B, \beta)$ are reversible $C^{*}$-dynamical systems. For any morphism $(A, \alpha) \stackrel{T}{\rightarrow}(B, \beta)$ the following conditions are equivalent:

i) $T$ is covariant,

ii) $\beta_{*} \circ T=T \circ \alpha_{*}$,

iii) $\bar{T}\left(\bar{\alpha}_{*}(1)\right) \leq \bar{\beta}_{*}(1)$,

iv) $\bar{T}\left(\bar{\alpha}_{*}(1)\right)=\bar{\beta}_{*}(1)$

Proof. Equivalences i) $\Leftrightarrow$ iii) $\Leftrightarrow$ iv) follow from Lemma 2.12. The implication ii) $\Rightarrow$ iv) is immediate. To close the cycle we show that i) $\Rightarrow$ ii). To this end recall that the mappings $A \ni a \mapsto\left(\alpha_{*} \circ \alpha\right)(a)=\bar{\alpha}_{*}(1) a \in(\operatorname{ker} \alpha)^{\perp}$ and $A \ni a \mapsto\left(\alpha \circ \alpha_{*}\right)(a)=\bar{\alpha}(1) a \bar{\alpha}(1) \in \bar{\alpha}(1) A \bar{\alpha}(1)$ are projections. Similarly, for $(B, \beta)$. Now using covariance of $T$ we get $T\left(\alpha_{*}(a)\right) \in(\operatorname{ker} \beta)^{\perp}$, for any $a \in A$. Therefore

$$
\begin{aligned}
T\left(\alpha_{*}(a)\right) & =\beta_{*}\left(\beta\left(T\left(\alpha_{*}(a)\right)\right)\right)=\beta_{*}\left(T\left(\alpha\left(\alpha_{*}(a)\right)\right)\right)=\beta_{*}(T(\bar{\alpha}(1) a \bar{\alpha}(1))) \\
& =\beta_{*}(\bar{\beta}(1) T(a) \bar{\beta}(1))=\beta_{*}(T(a)) .
\end{aligned}
$$

Lemma 2.14. Suppose that $(A, \alpha) \stackrel{T}{\rightarrow}(B, \beta)$ is an embedding and $(B, \beta)$ is a reversible $C^{*}$ dynamical system. Then $T$ is $J$-covariant where

$$
J=\left\{a \in A: \bar{\beta}_{*}(1) T(a)=T(a)\right\} .
$$

Proof. We have $\left\{a \in A: \bar{\beta}_{*}(1) T(a)=T(a)\right\}=\left\{a \in A: T(a) \in(\operatorname{ker} \beta)^{\perp}\right\}=T^{-1}\left((\operatorname{ker} \beta)^{\perp}\right)$.

Composition with covariant embeddings does not affect the covariance. 
B. K. KWAŚNIEWSKI

Lemma 2.15. Let $(B, \beta) \stackrel{S}{\rightarrow}(C, \gamma)$ be a covariant embedding. A morphism $(A, \alpha) \stackrel{T}{\rightarrow}(B, \beta)$ is covariant on $J$ iff $(A, \alpha) \stackrel{S \circ T}{\longrightarrow}(C, \gamma)$ is covariant on $J$.

Proof. We have $(S \circ T)^{-1}\left((\operatorname{ker} \gamma)^{\perp}\right)=T^{-1}\left(S^{-1}(\operatorname{ker} \gamma)^{\perp}\right)=T^{-1}\left((\operatorname{ker} \beta)^{\perp}\right)$.

We finish this subsection by noting that $C^{*}$-dyna is a category with direct limits.

Proposition 2.16. A direct sequence

$$
\left(B_{0}, \beta_{0}\right) \stackrel{T_{0}}{\longrightarrow}\left(B_{1}, \beta_{1}\right) \stackrel{T_{1}}{\longrightarrow}\left(B_{2}, \beta_{2}\right) \stackrel{T_{2}}{\longrightarrow} \ldots
$$

has a direct limit $(B, \beta)$ in the category $C^{*}$-dyna, where $B=\lim _{\longrightarrow}\left\{B_{n}, T_{n}\right\}$ is the $C^{*}$-algebraic direct limit and $\beta$ is the induced endomorphism.

Moreover, if all of the bonding maps $T_{n}, n \in \mathbb{N}$, are covariant morphism then the natural morphisms $\left(B_{n}, \beta_{n}\right) \stackrel{\phi_{n}}{\rightarrow}(B, \beta)$ are also covariant.

Proof. Let $n \in \mathbb{N}$. Notice first that the natural homomorphism $\phi_{n}: B_{n} \rightarrow B$ is non-degenerate. Indeed, any element in $B$ can be approximated by $\phi_{m}\left(b_{m}\right) \in B$ for certain $b_{m} \in B_{m}$ and $m>n$. As a composition of finite number of non-degenerate homomorphisms, the homomorphism $T_{m, n}:=T_{m} \circ \ldots \circ T_{n+1} \circ T_{n}: B_{n} \rightarrow B_{m}$ is non-degenerate. Thus $b_{m}=T_{m, n}\left(a_{n}\right) c_{m}$ for certain $a_{n} \in B_{n}, c_{m} \in B_{m}$. Hence $\phi_{m}\left(b_{m}\right)=\phi_{n}\left(a_{n}\right) \phi_{m}\left(c_{m}\right)$ and this proves our claim.

Clearly, the formula $\beta\left(\phi_{n}(b)\right):=\phi_{n}\left(\beta_{n}(b)\right), b \in B_{n}, n \in \mathbb{N}$, yields a well defined endomorphism $\beta: B \rightarrow B$. To see it is extendible let $\left\{\mu_{\lambda}\right\}$ be an approximate unit in $B_{0}$. As $\phi_{0}$ is nondegenerate, $\left\{\phi_{0}\left(\mu_{\lambda}\right)\right\}$ is an approximate unit in $B$. It follows that the (bounded and self-adjoint) net $\left\{\beta\left(\phi_{0}\left(\mu_{\lambda}\right)\right)\right\}$ converges strictly in $M(B)$ because for any $b \in B_{n}, n \in \mathbb{N}$, the net

$$
\beta\left(\phi_{0}\left(\mu_{\lambda}\right)\right) \phi_{n}(b)=\phi_{n}\left(T_{n, 0}\left(\beta_{0}\left(\mu_{\lambda}\right)\right) b\right)
$$

converges to $\phi_{n}\left(\bar{T}_{n, 0}\left(\bar{\beta}_{0}(1)\right) b\right)$. Hence $\beta$ is extendible and $(B, \beta) \in C^{*}$-dyna.

Let $(C, \gamma)$ be an arbitrary $C^{*}$-dynamical system equipped with morphisms $\left(B_{n}, \beta_{n}\right) \stackrel{\psi_{n}}{\rightarrow}(C, \gamma)$ such that $\psi_{n+1} \circ T_{n}=\psi_{n}$ for all $n \in \mathbb{N}$. Then the universal property of the $C^{*}$-algebraic direct limit $B$ implies that there is a homomorphism $\Psi: B \rightarrow C$ such that $\Psi \circ \phi_{n}=\psi_{n}, n \in \mathbb{N}$. In particular, $\Psi$ is non-degenerate because each of $\psi_{n}$ is. Since

$$
(\Psi \circ \beta) \circ \phi_{n}=\Psi \circ \phi_{n} \circ \beta_{n}=\psi_{n} \circ \beta_{n}=\gamma \circ \psi_{n}=(\gamma \circ \Psi) \circ \phi_{n},
$$

we conclude that $(B, \beta) \stackrel{\Psi}{\rightarrow}(C, \gamma)$ is a morphism, and $(B, \beta)$ is the direct limit in $C^{*}$-dyna.

Now suppose that all of the bonding maps $T_{m}, m \in \mathbb{N}$, are covariant and let $a \in\left(\operatorname{ker} \beta_{n}\right)^{\perp}$ for a fixed $n \in \mathbb{N}$. Covariance implies that for any $m>n$ we have $T_{m, n}(a) \in\left(\operatorname{ker} \beta_{m}\right)^{\perp}$. Now let $\phi_{k}(b) \in \operatorname{ker} \beta$ where $b \in B_{k}$ and $k>n$. For any $m>k$ we have $T_{m, n}(a) T_{m, k}(b) \in\left(\operatorname{ker} \beta_{m}\right)^{\perp}$ and $\phi_{m}\left(\beta_{m}\left(T_{m, n}(a) T_{m, k}(b)\right)\right)=0$. Using these relations and the explicit formula for norm in the direct limit $B$ we get

$$
\begin{aligned}
\left\|\phi_{n}(a) \phi_{k}(b)\right\| & =\lim _{m \rightarrow \infty}\left\|T_{m, n}(a) T_{m, k}(b)\right\|=\lim _{m \rightarrow \infty}\left\|\beta_{m}\left(T_{m, n}(a) T_{m, k}(b)\right)\right\| \\
& =\lim _{m \rightarrow \infty}\left\|\phi_{m}\left(\beta_{m}\left(T_{m, n}(a) T_{m, k}(b)\right)\right)\right\|=0 .
\end{aligned}
$$

Hence $\phi_{n}(a) \phi_{k}(b)=0$ and this implies that $\phi_{n}(a) \in(\operatorname{ker} \beta)^{\perp}$. Accordingly, $\left(B_{n}, \beta_{n}\right) \stackrel{\phi_{n}}{\rightarrow}(B, \beta)$ is covariant.

Remark 2.17. The direct limit system $(B, \beta)$ sits in both its strictly continuous extension $(M(B), \bar{\beta})$ and the direct limit system associated to the direct sequence obtained by strictly continuous extensions:

$$
\left(M\left(B_{0}\right), \bar{\beta}_{0}\right) \stackrel{\bar{T}_{0}}{\longrightarrow}\left(M\left(B_{1}\right), \bar{\beta}_{1}\right) \stackrel{\bar{T}_{1}}{\longrightarrow}\left(M\left(B_{2}\right), \bar{\beta}_{2}\right) \stackrel{\bar{T}_{2}}{\longrightarrow} \ldots
$$

In fact, with obvious identifications we have $B \subseteq \lim _{\longrightarrow}\left\{M\left(B_{n}\right), \bar{T}_{n}\right\} \subseteq M(B)$. 


\section{NATURAL REVERSIBLE EXTENSIONS OF $C^{*}$-DYNAMICAL SYSTEMS AND INVARIANT IDEALS}

We fix a $C^{*}$-dynamical system $(A, \alpha)$ and an ideal $J$ in $(\operatorname{ker} \alpha)^{\perp}$. Our first aim is to construct a universal reversible $C^{*}$-dynamical system $(B, \beta)$ which is a $J$-extension of $(A, \alpha)$. Next we study relationship between invariant ideals in $(B, \beta)$ and $(A, \alpha)$. In the sequel, we will abbreviate the long phrase 'reversible $C^{*}$-dynamical system which is a $J$-extension' to the short expression 'reversible J-extension'.

3.1. The main construction. We will define the system $(B, \beta)$ as a direct limit of certain approximating $C^{*}$-dynamical systems $\left(B_{n}, \beta_{n}\right), n \in \mathbb{N}$, that are constructed as follows. Denote by $q: A \rightarrow A / J$ the quotient map and for each $n \in \mathbb{N}$ put

$$
A_{n}:=\bar{\alpha}^{n}(1) A \bar{\alpha}^{n}(1) .
$$

Define the $C^{*}$-algebra $B_{n}$ as a direct sum of the form

$$
B_{n}=q\left(A_{0}\right) \oplus q\left(A_{1}\right) \oplus \ldots \oplus q\left(A_{n-1}\right) \oplus A_{n} .
$$

Let $\beta_{n}: B_{n} \rightarrow B_{n}$ be given by

$$
\beta_{n}\left(a_{0} \oplus a_{1} \oplus \ldots \oplus a_{n}\right)=a_{1} \oplus a_{2} \oplus \ldots \oplus q\left(a_{n}\right) \oplus \alpha\left(a_{n}\right) .
$$

Clearly, $\beta_{n}$ is an extendible endomorphism. Thus we have a sequence $\left(B_{n}, \beta_{n}\right), n \in \mathbb{N}$, of $C^{*}$-dynamical systems with $\left(B_{0}, \beta_{0}\right)=(A, \alpha)$.

Define the bonding homomorphisms $T_{n}: B_{n} \rightarrow B_{n+1}, n \in \mathbb{N}$, as schematically presented by the diagram

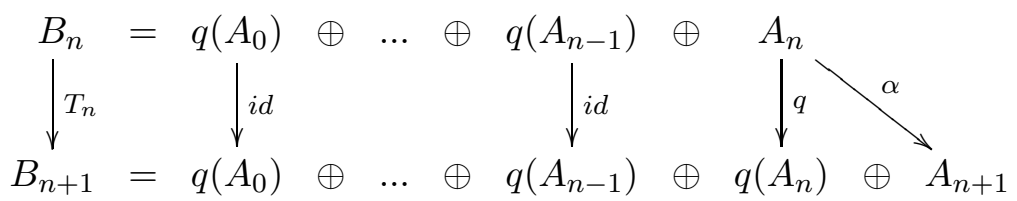

and formally given by the formula

$$
T_{n}\left(a_{0} \oplus \ldots \oplus a_{n-1} \oplus a_{n}\right)=a_{0} \oplus \ldots \oplus a_{n-1} \oplus q\left(a_{n}\right) \oplus \alpha\left(a_{n}\right),
$$

where $a_{k} \in q\left(A_{k}\right), k=0, \ldots, n-1$, and $a_{n} \in A_{n}$. Since $J \subseteq(\operatorname{ker} \alpha)^{\perp}$, the homomorphisms $T_{n}$ are injective. Plainly, $T_{n} \circ \beta_{n}=\beta_{n+1} \circ T_{n}, n \in \mathbb{N}$. Hence we have the direct sequence of embeddings:

$$
(A, \alpha)=\left(B_{0}, \beta_{0}\right) \stackrel{T_{0}}{\longrightarrow}\left(B_{1}, \beta_{1}\right) \stackrel{T_{1}}{\longrightarrow}\left(B_{2}, \beta_{2}\right) \stackrel{T_{2}}{\longrightarrow} \ldots
$$

Theorem 3.1. Let $(B, \beta)$ be the direct limit of the direct sequence (11). Then $(B, \beta)$ is a reversible $J$-extension of $(A, \alpha)$, with an embedding $T$, possessing the following properties:

i) $B=\sum_{n=0}^{\infty} \beta_{*}^{n}(T(A))$ and $J=\left\{a \in A: \bar{\beta}_{*}(1) T(a)=T(a)\right\}$.

ii) If $(C, \gamma)$ is a reversible $J$-extension of $(A, \alpha)$ and $S$ is the corresponding embedding, then there is a unique covariant embedding $\widetilde{S}$ of $(B, \beta)$ into $(C, \gamma)$ such that $S=\widetilde{S} \circ T$, i.e. the diagram

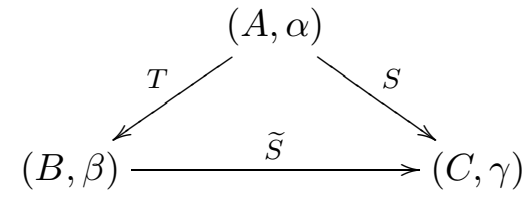

commutes.

iii) Any reversible extension of $(A, \alpha)$ that possess one of the properties of $(B, \beta)$ described in item i) or item ii) is equivalent to $(B, \beta)$. 
B. K. KWAŚNIEWSKI

Proof. Let $n>0$. We have $\left(\operatorname{ker} \beta_{n}\right)^{\perp}=0 \oplus q\left(A_{1}\right) \oplus \ldots \oplus q\left(A_{n-1}\right) \oplus A_{n}$. Thus by the form of the bonding map $T_{n}$, we see that $\left(B_{n}, \beta_{n}\right) \stackrel{T_{n}}{\longrightarrow}\left(B_{n+1}, \beta_{n+1}\right)$ is a covariant embedding and clearly $(A, \alpha) \stackrel{T_{0}}{\longrightarrow}\left(B_{1}, \beta_{1}\right)$ is a $J$-embedding. Accordingly, denoting by $\phi_{n}: B_{n} \rightarrow B$ the natural embeddings into the direct limit $B$, we deduce from Lemma 2.15] and Proposition 2.16, that $T:=\phi_{0}$ is a $J$-embedding of $(A, \alpha)$ into $(B, \beta)$.

To prove that $(B, \beta)$ is reversible, recall that we may treat $\lim _{\longrightarrow}\left\{M\left(B_{n}\right), \bar{T}_{n}\right\}$ as a $C^{*}$-subalgebra of $M(B)$, cf. Remark 2.17. In particular, for each $n>0$ the projection from $B_{n}$ onto $\left(\operatorname{ker} \beta_{n}\right)^{\perp}$, which we denote by $p_{n}$, is an element of $M\left(B_{n}\right)$, and $\bar{T}_{n}\left(p_{n}\right)=p_{n+1}$. It follows that $\bar{\phi}_{n}\left(p_{n}\right)$ is the projection from $B$ onto $(\operatorname{ker} \beta)^{\perp}$. Hence $(\operatorname{ker} \beta)^{\perp}$ is a complemented ideal in $B$. Similarly, for each $n>0$ we have $\bar{T}_{n}\left(\bar{\beta}_{n}(1)\right)=\bar{\beta}_{n+1}(1)$, and therefore $\bar{\beta}(1)=\bar{\phi}_{n}\left(\bar{\beta}_{n}(1)\right)$. To see that $\beta(B)$ is a hereditary subalgebra it suffices to note that $\bar{\beta}(1) B \bar{\beta}(1) \subseteq \beta(B)$. To show the latter put $E_{p}(a):=p a p$, for any $a, p \in M(A)$. Then

$$
\bar{\beta}(1)\left(\phi_{n}\left(q\left(a_{0}\right) \oplus \ldots \oplus q\left(a_{n-1}\right) \oplus a_{n}\right)\right) \bar{\beta}(1)
$$

equals to

$$
\phi_{n}\left(q\left(E_{\bar{\alpha}(1)}\left(a_{0}\right)\right) \oplus \ldots \oplus q\left(E_{\bar{\alpha}^{n}(1)}\left(a_{n-1}\right)\right) \oplus E_{\bar{\alpha}^{n+1}(1)}\left(a_{n}\right)\right),
$$

which in turn equals to

$$
\beta\left(\phi_{n+1}\left(0 \oplus q\left(E_{\bar{\alpha}(1)}\left(a_{0}\right)\right) \oplus \ldots \oplus q\left(E_{\bar{\alpha}^{n}(1)}\left(a_{n-1}\right)\right) \oplus E_{\bar{\alpha}^{n+1}(1)}\left(a_{n}\right)\right)\right) .
$$

Thus $\beta(B)=\bar{\beta}(1) B \bar{\beta}(1)$ and by Proposition 2.3 the system $(B, \beta)$ is reversible. Moreover, if follows from the above calculation and (6) that the complete transfer operator $\beta_{*}$ maps $\phi_{n}\left(B_{n}\right)$ into $\phi_{n+1}\left(B_{n+1}\right)$ where

$$
\beta_{*}\left(\left(\phi_{n}\left(q\left(a_{0}\right) \oplus \ldots \oplus q\left(a_{n-1}\right) \oplus a_{n}\right)\right)\right)
$$

equals to

$$
\phi_{n+1}\left(0 \oplus q\left(E_{\bar{\alpha}(1)}\left(a_{0}\right)\right) \oplus \ldots \oplus q\left(E_{\bar{\alpha}^{n}(1)}\left(a_{n-1}\right)\right) \oplus E_{\bar{\alpha}^{n+1}(1)}\left(a_{n}\right)\right) .
$$

This proves the initial part of the assertion.

i). Recall that $T=\phi_{0}$. Using the above description of $\beta_{*}$ one checks that for $b_{k} \in A_{k}$, $k=0, \ldots, n$, we have

$$
\left(\beta_{*}^{k} \circ T\right)\left(b_{k}\right)=\phi_{n}\left(0 \oplus \ldots \oplus 0 \oplus q\left(b_{k}\right) \oplus q\left(\alpha\left(b_{k}\right)\right) \oplus \ldots q\left(\alpha^{n-k-1}\left(b_{k}\right)\right) \oplus \alpha^{n-k}\left(a_{n}\right)\right) .
$$

Thus putting $b_{k}=a_{k}-\sum_{i=0}^{k-1} \alpha^{k-i}\left(a_{i}\right)$ where $a_{k} \in A_{k}, k=0, \ldots, n$, and summing over $k$ we get

$$
\phi_{n}\left(q\left(a_{0}\right) \oplus \ldots \oplus q\left(a_{n-1}\right) \oplus a_{n}\right)=\sum_{k=0}^{n}\left(\beta_{*}^{k} \circ T\right)\left(a_{k}-\sum_{i=0}^{k-1} \alpha^{k-i}\left(a_{i}\right)\right) .
$$

Hence $B \subseteq \sum_{n=0}^{\infty} \beta_{*}^{n}(T(A))$. As the reverse inclusion is obvious we get $B=\sum_{n=0}^{\infty} \beta_{*}^{n}(T(A))$. We have $J=\left\{a \in A: \bar{\beta}_{*}(1) T(a)=T(a)\right\}$ by Lemma 2.14 .

ii). We claim that the desired covariant embedding $(B, \beta) \stackrel{\widetilde{S}}{\rightarrow}(C, \gamma)$ can be defined by the formula

$$
\widetilde{S}\left(\phi_{n}\left(q\left(a_{0}\right) \oplus q\left(a_{1}\right) \oplus \ldots \oplus a_{n}\right)\right)=Q S\left(a_{0}\right)+\gamma_{*}\left(Q S\left(a_{1}\right)\right) \ldots+\gamma_{*}^{n}\left(S\left(a_{n}\right)\right)
$$

where $Q:=\left(1-\bar{\gamma}_{*}(1)\right)$ is a central projection in $M(C)$, see Proposition 2.6. To this end notice that $\bar{S}\left(\bar{\alpha}^{k}(1)\right)=\bar{\gamma}^{k}(1), k \in \mathbb{N}$, and $J=\left\{a \in A: \bar{\gamma}_{*}(1) S(a)=S(a)\right\}$, cf. Lemma 2.14. Thus one deduces that

$$
A_{k} / J \ni q(a) \longmapsto Q S(a) \in Q \bar{\gamma}^{k}(1) S(A) \bar{\gamma}^{k}(1)
$$


is a well defined isomorphism. Moreover, composing it with the isomorphism $\gamma_{*}^{k}: \gamma^{k}(C)=$ $\bar{\gamma}^{k}(1) C \bar{\gamma}^{k}(1) \rightarrow \bar{\gamma}_{*}^{k}(1) C$ we get the injective homomorphism

$$
A_{k} / J \ni q(a) \longmapsto \gamma_{*}^{k}(Q S(a)) \in \bar{\gamma}_{*}^{k}(Q) C .
$$

Similarly, for each $n \in \mathbb{N}$, we get the injective homomorphism

$$
A_{n} \ni a \longmapsto \gamma_{*}^{n}(S(a)) \in \bar{\gamma}_{*}^{n}(1) C .
$$

Now, since the projections $\left\{\bar{\gamma}_{*}^{k}(1)\right\}_{k \in \mathbb{N}}$ are decreasing and central in $M(C)$ we see that the projections

$$
\bar{\gamma}_{*}^{k}(Q)=\bar{\gamma}_{*}^{k}(1)-\bar{\gamma}_{*}^{k+1}(1), k=0, \ldots, n-1, \quad \text { and } \quad \bar{\gamma}_{*}^{n}(1)
$$

are pair-wise orthogonal and central in $M(C)$. Hence the direct sum of mappings (13) for $k=0,1 \ldots, n-1$ and the mapping (14), establishes an injective homomorphism from $B_{n}=$ $\bigoplus_{k=0}^{n-1} q\left(A_{k}\right) \oplus A_{n}$ to $C=\bigoplus_{k=0}^{n-1} \bar{\gamma}_{*}^{k}(Q) C \oplus \bar{\gamma}_{*}^{n}(1) C$. As $\phi_{n}: B_{n} \rightarrow B$ is injective, we conclude that the formula (12) yields a well defined injective homomorphism from $\phi_{n}\left(B_{n}\right)$ into $C$. To see that it actually defines a homomorphism from $B$ to $C$ note that

$$
S\left(a_{n}\right)=Q S\left(a_{n}\right)+\bar{\gamma}_{*}(1) S\left(a_{n}\right)=Q S\left(a_{n}\right)+\gamma_{*}\left(\gamma\left(S\left(a_{n}\right)\right)=Q S\left(a_{n}\right)+\gamma_{*}\left(S\left(\alpha\left(a_{n}\right)\right),\right.\right.
$$

and hence by (12), we get

$$
\begin{aligned}
\widetilde{S}\left(\phi_{n}\left(q\left(a_{0}\right) \oplus \ldots \oplus a_{n}\right)\right) & =\widetilde{S}\left(\phi_{n+1}\left(q\left(a_{0}\right) \oplus \ldots \oplus q\left(a_{n}\right) \oplus \alpha\left(a_{n}\right)\right)\right) \\
& =\widetilde{S}\left(\phi_{n+1}\left(T_{n}\left(q\left(a_{0}\right) \oplus \ldots \oplus a_{n}\right)\right)\right) .
\end{aligned}
$$

Hence $\widetilde{S}: B \rightarrow C$ is an injective homomorphism.

Clearly, $S=\widetilde{S} \circ T$. In particular, $\widetilde{S}: B \rightarrow C$ is non-degenerate because $S: A \rightarrow C$ is. Furthermore, for $a \in \bar{\gamma}^{k}(1) C \bar{\gamma}^{k}(1), k>0$, we have

$$
\left.\gamma\left(\gamma_{*}^{k}(a)\right)\right)=\bar{\gamma}(1) \gamma_{*}^{k-1}(a) \bar{\gamma}(1)=\gamma_{*}^{k-1}\left(\bar{\gamma}^{k}(1) a \bar{\gamma}^{k}(1)\right)=\gamma_{*}^{k-1}(a) .
$$

Therefore, in view of (12), since $\gamma(Q)=0$, we get

$$
\begin{aligned}
\gamma\left(\widetilde{S}\left(\phi_{n}\left(q\left(a_{0}\right) \oplus q\left(a_{1}\right) \oplus \ldots \oplus a_{n}\right)\right)\right) & =S\left(Q a_{1}\right)+\gamma_{*}\left(Q S\left(a_{2}\right)\right)+\ldots+\gamma_{*}^{n-1}\left(S\left(a_{n}\right)\right) \\
& =\widetilde{S}\left(\beta\left(\phi_{n}\left(a_{0} \oplus a_{1} \oplus \ldots \oplus a_{n}\right)\right)\right) .
\end{aligned}
$$

Hence $\gamma \circ \widetilde{S}=\widetilde{S} \circ \beta$ and $(B, \beta) \stackrel{\widetilde{S}}{\rightarrow}(C, \gamma)$ is an embedding. Moreover, since

$$
\overline{\widetilde{S}}\left(\bar{\beta}_{*}(1)\right)=\overline{\widetilde{S}}\left(\bar{\phi}_{1}(0 \oplus \bar{\alpha}(1))\right)=\bar{\gamma}_{*}(\bar{S}(\bar{\alpha}(1)))=\bar{\gamma}_{*}(\bar{\gamma}(1))=\bar{\gamma}_{*}(1),
$$

$\widetilde{S}$ is covariant by Lemma 2.13 .

This proves the existence part. Uniqueness of $\widetilde{S}$ follows immediately from the relations $B=$ $\sum_{n=0}^{\infty} \beta_{*}^{n}(T(A))$ and $\gamma_{*} \circ \widetilde{S}=\widetilde{S} \circ \beta_{*}$, cf. Lemma 2.13 ,

iii). Suppose $(A, \alpha) \stackrel{S}{\rightarrow}(C, \gamma)$ is an embedding of $(A, \alpha)$ into a reversible $C^{*}$-dynamical system $(C, \gamma)$. If $C=\sum_{n=0}^{\infty} \gamma_{*}^{n}(S(A))$ and $J=\left\{a \in A: \bar{\gamma}_{*}(1) S(a)=S(a)\right\}$, then $S$ is a $J$-embedding, see Lemma 2.14, and in view of item i) the embedding $(B, \beta) \stackrel{\widetilde{S}}{\rightarrow}(C, \gamma)$ whose existence is guaranteed by item ii) is surjective. Hence $(B, \beta)$ and $(C, \gamma)$ are equivalent.

If $(C, \gamma)$ possess the property described in item ii) then there is a covariant embedding $(C, \gamma) \stackrel{\widetilde{T}}{\rightarrow}$ $(B, \beta)$ such that $T=\widetilde{T} \circ S$. Appealing again to Lemma 2.13 we get

$$
\widetilde{T} \circ \gamma_{*} \circ S=\beta_{*} \circ \widetilde{T} \circ S=\beta_{*} \circ T \text {. }
$$

Thus by item i) the embedding $\widetilde{T}$ is onto $B$. Consequently, $(B, \beta)$ and $(C, \gamma)$ are equivalent.

We extend [29, Definition 3.4] to non-unital case as follows. 
B. K. KWAŚNIEWSKI

Definition 3.2. Let $(A, \alpha)$ be a $C^{*}$-dynamical system, $J$ an ideal in $(\operatorname{ker} \alpha)^{\perp}$ and $(B, \beta)$ the direct limit of (11). We call $(B, \beta)$ the natural reversible $J$-extension of $(A, \alpha)$. If $J=(\operatorname{ker} \alpha)^{\perp}$ we drop the prefix ' $J-$ '.

Remark 3.3. In the sequel we will usually identify $A$ with the corresponding subalgebra of $B$. Then the natural reversible $J$-extension $(B, \beta)$ can be characterized as a reversible $C^{*}$ dynamical system satisfying

$$
B=\sum_{n=0}^{\infty} \beta_{*}^{n}(A), \quad \overline{\beta_{*}(1)} A \cap A=J,\left.\quad \beta\right|_{A}=\alpha .
$$

To extend the above picture, so that it covers also the algebras $B_{n}, n \in \mathbb{N}$, we put $q:=1-\overline{\beta_{*}(1)}$ (this makes a perfect match with our previous notation on the quotient map $A \rightarrow A / J)$. We identify the algebra $B_{n}, n \in \mathbb{N}$, with its image in $B$ by the mapping

$$
B_{n} \ni \bigoplus_{k=0}^{n} q\left(a_{k}\right) \oplus a_{n} \longmapsto \sum_{k=0}^{n-1} \beta_{*}^{k}\left(q a_{k}\right)+\beta_{*}^{n}\left(a_{n}\right) \in B .
$$

With this identification we have

$$
B_{n}=\sum_{k=0}^{n-1} \beta_{*}^{k}\left(q A_{k}\right)+\beta_{*}^{n}\left(A_{n}\right)=\sum_{k=0}^{n-1} \beta_{*}^{k}(q) \beta_{*}^{k}(A)+\beta_{*}^{n}(A)=\sum_{k=0}^{n-1} \beta_{*}^{k}(q) B+\beta_{*}^{n}(A) .
$$

The above sums are actually direct sums of $C^{*}$-algebras because the projections $\bar{\beta}_{*}^{n}(1)$ and $\bar{\beta}_{*}^{k}(q)=\bar{\beta}_{*}^{k}(1)-\bar{\beta}_{*}^{k+1}(1), k=0, \ldots, n-1$, are pairwise orthogonal and central in $M(B)$, cf. Proposition 2.6.

3.2. Invariant ideals and $J$-pairs. Now we introduce invariant ideals and discuss the relevant notions and facts that will lead us to description of such ideals in natural reversible extensions defined in the previous subsection.

Definition 3.4. We say that an ideal $I$ in $A$ is a positively invariant ideal in $(A, \alpha)$ if $\alpha(I) \subseteq I$. Any such ideal $I$ induces a quotient $C^{*}$-dynamical system $\left(A / I, \alpha_{I}\right)$ where $\alpha_{I}(a+I)=\alpha(a)+I$ for all $a \in A$. We will refer to $\left(A / I, \alpha_{I}\right)$ as a subsystem of $(A, \alpha)$.

When regarding negative invariance, as in the case of extensions, it is useful to consider a family of such notions parametrized by an ideal.

Definition 3.5. Let $I$ and $J$ be ideals in $A$ where $J \subseteq(\operatorname{ker} \alpha)^{\perp}$. We say that $I$ is $J$-negatively invariant ideal in $(A, \alpha)$ if $J \cap \alpha^{-1}(I) \subseteq I$. If $I$ is both positively invariant and $J$-negatively invariant we say that $I$ is $J$-invariant. If $J=(\operatorname{ker} \alpha)^{\perp}$ we drop the prefix ' $J-$ '.

When $\alpha$ is an automorphism invariance of $I$ means that $\alpha(I)=I$. More generally, we have

Lemma 3.6. Suppose $(A, \alpha)$ is a reversible $C^{*}$-dynamical system and let $I$ be an ideal in $A$. The following conditions are equivalent:

i) $I$ is invariant in $(A, \alpha)$,

ii) $\alpha(I) \subseteq I$ and $\alpha_{*}(I) \subseteq I$,

iii) $\alpha(I)=\bar{\alpha}(1) I \bar{\alpha}(1)$,

iv) $\alpha_{*}(I)=\bar{\alpha}_{*}(1) I$.

In particular, if $I$ is invariant the system $\left(A / I, \alpha_{I}\right)$ is reversible and the set of invariant ideals in $(A, \alpha)$, ordered by inclusion, is a lattice.

Proof. i) $\Leftrightarrow$ ii). By the form of the complete transfer operator, see (6), we have $\alpha^{-1}(I)=$ $\operatorname{ker} \alpha \oplus \alpha_{*}(I)$. Thus negative invariance of $I$ is equivalent to the inclusion $\alpha_{*}(I) \subseteq I$. 
ii $\Rightarrow$ iii). Inclusion $\alpha(I) \subseteq I$ implies that $\alpha(I) \subseteq \bar{\alpha}(1) I \bar{\alpha}(1)$, while both of the inclusions in ii) imply that $\bar{\alpha}(1) I \bar{\alpha}(1)=\alpha\left(\alpha_{*}(I)\right) \subseteq I$.

iii) $\Rightarrow$ iv). We have $\alpha_{*}(I)=\alpha_{*}(\bar{\alpha}(1) I \bar{\alpha}(1))=\alpha_{*}(\alpha(I))=\bar{\alpha}_{*}(1) I$.

iv) $\Rightarrow$ ii). We have $\alpha(I)=\alpha\left(\bar{\alpha}_{*}(1) I\right)=\alpha\left(\alpha_{*}(I)\right)=\bar{\alpha}(1) I \bar{\alpha}(1) \subseteq I$ and $\alpha_{*}(I)=\bar{\alpha}_{*}(1) I \subseteq I$. The remaining part of the assertion is now straightforward.

Note that an ideal $I$ is $J$-invariant if and only if the system $\left(A / I, \alpha_{I}\right)$ is well defined and $q_{I}(J) \subseteq\left(\operatorname{ker} \alpha_{I}\right)^{\perp}$. Thus $J$-invariant ideals correspond to subsystems of $(A, \alpha)$ which 'admit $J$-extensions'. Alternatively, $J$-invariant ideals in $(A, \alpha)$ can be viewed as parts of invariant ideals in $J$-extensions of $(A, \alpha)$.

Lemma 3.7. Let $T$ be a $J$-embedding of $(A, \alpha)$ into a certain $C^{*}$-dynamical system $(B, \beta)$. If $\widetilde{I}$ is an invariant ideal in in $(B, \beta)$ then

$$
I:=T^{-1}(\widetilde{I})
$$

is a $J$-invariant ideal in $(A, \alpha)$. In particular, $T$ factors through to an $q_{I}\left(I^{\prime}\right)$-embedding $\left(A / I, \alpha_{I}\right) \stackrel{T_{\widetilde{I}}}{\longrightarrow}\left(B / \widetilde{I}, \beta_{\widetilde{I}}\right)$ where $I^{\prime}$ is an ideal in $A$ such that

$$
J \subseteq I^{\prime} \quad \text { and } \quad I^{\prime} \cap \alpha^{-1}(I)=I .
$$

If $(B, \beta)$ is reversible then $I^{\prime}=\left\{a \in A:\left(1-\bar{\beta}_{*}(1)\right) T(a) \in \widetilde{I}\right\}$.

Proof. Since $T(\alpha(I))=\beta(T(I))=\beta\left(T\left(T^{-1}(\widetilde{I})\right)\right) \subseteq \beta(\widetilde{I}) \subseteq \widetilde{I}$ we have $\alpha(I) \subseteq I$. To show the negative $J$-invariance of $I$ note first that

$$
T\left(\alpha^{-1}(I)\right)=T\left((T \circ \alpha)^{-1}(\widetilde{I})\right)=T\left((\beta \circ T)^{-1}(\widetilde{I})\right)=T\left(T^{-1}\left(\beta^{-1}(\widetilde{I})\right)\right) \subseteq \beta^{-1}(\widetilde{I}) .
$$

Therefore by negative invariance of $\widetilde{I}$ we have

$$
T\left(J \cap \alpha^{-1}(I)\right) \subseteq(\operatorname{ker} \beta)^{\perp} \cap T\left(\alpha^{-1}(I)\right) \subseteq(\operatorname{ker} \beta)^{\perp} \cap \beta^{-1}(\widetilde{I}) \subseteq \widetilde{I} .
$$

Hence $J \cap \alpha^{-1}(I) \subseteq I$, and $I$ is $J$-invariant. Plainly, $T_{\widetilde{I}}(a+I)=T(a)+\widetilde{I}$ defines an embedding of the subsystem $\left(A / I, \alpha_{I}\right)$ into $\left(B / \widetilde{I}, \beta_{\widetilde{I}}\right)$. By definition $T_{\widetilde{I}}$ is $T_{\widetilde{I}}^{-1}\left(\left(\operatorname{ker} \beta_{\widetilde{I}}\right)^{\perp}\right)$-covariant. Letting $I^{\prime}:=q_{I}^{-1}\left(T_{\widetilde{I}}^{-1}\left(\left(\operatorname{ker} \beta_{\widetilde{I}}\right)^{\perp}\right)\right)$ we obtain the assertion. In particular, since $\alpha^{-1}(I)=q_{I}^{-1}\left(\operatorname{ker} \alpha_{I}\right)=$ $q_{I}^{-1}\left(T_{\widetilde{I}}^{-1}\left(\operatorname{ker} \beta_{\widetilde{I}}\right)\right)$, the relations

$$
J \cap \alpha^{-1}(I) \subseteq I, \quad T_{\widetilde{I}}^{-1}\left(\left(\operatorname{ker} \beta_{\widetilde{I}}\right)^{\perp}\right) \cap \operatorname{ker} \alpha_{I}=\{0\}
$$

imply (17).

Suppose now that $(B, \beta)$ is reversible and note that by Lemma 3.6 also the subsystem $\left(B / \widetilde{I}, \beta_{\widetilde{I}}\right)$ is reversible; its complete transfer operator is given by $\beta_{* \widetilde{I}}(b+\widetilde{I})=\beta_{*}(b)+\widetilde{I}, b \in B$. Hence applying Lemma 2.14 to the embedding $T_{\widetilde{I}}$ we get

$$
\begin{aligned}
a \in I^{\prime} & \Longleftrightarrow q_{I}(a) \in T_{\widetilde{I}}^{-1}\left(\left(\operatorname{ker} \beta_{\widetilde{I}}\right)^{\perp}\right) \Longleftrightarrow \bar{\beta}_{*, \widetilde{I}}(1) T_{\widetilde{I}}\left(q_{I}(a)\right)=T_{\widetilde{I}}\left(q_{I}(a)\right) \\
& \Longleftrightarrow \bar{\beta}_{*}(1) T(a)+\widetilde{I}=T(a)+\widetilde{I} \Longleftrightarrow\left(1-\bar{\beta}_{*}(1)\right) T(a) \in \widetilde{I} .
\end{aligned}
$$

Remark 3.8. In general the ideal $I^{\prime}$ above carries an essential data concerning the ideal $\widetilde{I}$, which is not visible from the standpoint of $I$ and $J$. Indeed, when $I$ is a $J$-invariant ideal then $I+J$ is the minimal ideal satisfying (17). On one hand, in the favorable situation when $A=\operatorname{ker} \alpha \oplus(\operatorname{ker} \alpha)^{\perp}$ and $J=(\operatorname{ker} \alpha)^{\perp}$, any ideal satisfying (17) has to be of the form $I^{\prime}=I+J$ (as we then have $\left.I^{\prime}=\left(I^{\prime} \cap \operatorname{ker} \alpha\right) \oplus\left(I^{\prime} \cap(\operatorname{ker} \alpha)^{\perp}\right) \subseteq I+J\right)$. On the other hand, as the following example shows when ker $\alpha$ is not complemented in $A$ there might be ideals $I^{\prime}$ satisfying (17) that are strictly larger than $I+J$. 
Example 3.9. Let $A=C([0,1])$ and $\alpha(a)=a \circ \varphi$ where $\varphi:[0,1] \rightarrow[0,1]$ is given by $\varphi(x):=x / 2$. Put $J=(\operatorname{ker} \alpha)^{\perp}=C_{0}\left(\left[0, \frac{1}{2}\right)\right)$ and let $I$ be the ideal $C_{0}([0,1] \backslash K)$ where $K=$ $\left\{0,1, \frac{1}{2}, \frac{1}{4}, \frac{1}{8}, \ldots\right\}$. Since $\alpha(I)=C_{0}\left([0,1] \backslash \varphi^{-1}(K)\right)=I$ and $\alpha^{-1}(I)=C_{0}([0,1] \backslash \varphi(K)) \subseteq I$, the ideal $I$ is positively invariant and both of the ideals

$$
I_{1}^{\prime}:=C_{0}([0,1)), \quad I_{2}^{\prime}:=C_{0}([0,1) \backslash\{1 / 2\})
$$

satisfy relations (17). Actually these are the only such ideals. The only difference between $I_{1}^{\prime}$ and $I_{2}^{\prime}$ is 'located' in the boundary $\{1 / 2\}$ of the hull of $\operatorname{ker} \alpha$ (which is not empty because ker $\alpha$ is not complemented in $A$ ).

The above remark and example lead us to the following definition.

Definition 3.10. Let $I, I^{\prime}, J$ be ideals in $A$ where $J \subseteq(\operatorname{ker} \alpha)^{\perp}$. We say that $\left(I, I^{\prime}\right)$ is a $J$-pair for $(A, \alpha)$ if

$$
I \text { is positively invariant, } J \subseteq I^{\prime} \text { and } I^{\prime} \cap \alpha^{-1}(I)=I .
$$

Note that then $I$ is automatically $J$-invariant. We equip the set of $J$-pairs for $(A, \alpha)$ with a natural partial order induced by inclusion: $\left(I_{1}, I_{1}^{\prime}\right) \subseteq\left(I_{2}, I_{2}^{\prime}\right) \stackrel{\text { def }}{\Longleftrightarrow} I_{1} \subseteq I_{2}$ and $I_{1}^{\prime} \subseteq I_{2}^{\prime}$.

$J$-pairs arise naturally from morphisms.

Lemma 3.11. A morphism $(A, \alpha) \stackrel{T}{\rightarrow}(B, \beta)$ is covariant on $J \subseteq(\operatorname{ker} \alpha)^{\perp}$ if and only if

$$
I:=\operatorname{ker} T \quad \text { and } \quad I^{\prime}:=T^{-1}\left((\operatorname{ker} \beta)^{\perp}\right)
$$

form a $J$-pair $\left(I, I^{\prime}\right)$.

Proof. Since $I=T^{-1}(\{0\})$ is positively invariant and $I \subseteq I^{\prime}$ we have $I \subseteq I^{\prime} \cap \alpha^{-1}(I)$. The reverse inclusion holds because for $a \in I^{\prime} \cap \alpha^{-1}(I)$ we have $T(a) \in(\operatorname{ker} \beta)^{\perp}$ and $\beta(T(a))=$ $T(\alpha(a))=0$, which implies that $a \in \operatorname{ker} T=I$. Hence $I^{\prime} \cap \alpha^{-1}(I)=I$. Thus $\left(I, I^{\prime}\right)$ is a $J$-pair if and only if $T$ is covariant on $J$.

3.3. Invariant ideals in natural reversible extensions. Now we are in a position to provide the description of invariant ideals in natural reversible extensions.

Theorem 3.12. Let $(B, \beta)$ be a natural reversible $J$-extension of $(A, \alpha)$ and retain the identifications and notation from Remark [3.3. We have an order preserving bijection between J-pairs $\left(I, I^{\prime}\right)$ for $(A, \alpha)$ and invariant ideals $\widetilde{I}$ in $(B, \beta)$, given by

$$
\begin{gathered}
I=\widetilde{I} \cap A, \quad I^{\prime}=\{a \in A: q a \in \widetilde{I}\}, \\
\widetilde{I} \cap B_{n}=\sum_{k=0}^{n-1} \beta_{*}^{k}\left(q I^{\prime}\right)+\beta_{*}^{n}(I), \quad n \in \mathbb{N} .
\end{gathered}
$$

In particular, the set of $J$-pairs is a lattice. Moreover, denoting by $\left(B_{\left(I, I^{\prime}\right)}, \beta_{\left(I, I^{\prime}\right)}\right)$ the natural reversible $q_{I}\left(I^{\prime}\right)$-extension of the subsystem $\left(A_{I}, \alpha_{I}\right)$ we have

$$
\left(B / \widetilde{I}, \beta_{\widetilde{I}}\right) \cong\left(B_{\left(I, I^{\prime}\right)}, \beta_{\left(I, I^{\prime}\right)}\right)
$$

where $\widetilde{I}$ is the invariant ideal corresponding to a $J$-pair $\left(I, I^{\prime}\right)$.

Proof. Let $\widetilde{I}$ be an invariant ideal in $(B, \beta)$. Lemma 3.7 tells us that (18) defines a $J$-pair $\left(I, I^{\prime}\right)$. To show that $\widetilde{I}$ is determined by $\left(I, I^{\prime}\right)$ it suffices to verify (19). But the latter is simple. Indeed, taking into account the direct sum decomposition (15), we get that the sum $\sum_{k=0}^{n-1} \beta_{*}^{k}\left(q a_{k}\right)+\beta_{*}^{n}\left(a_{n}\right)$, where $a_{k} \in A_{k}$, is in $\widetilde{I}$ if and only if its summands are in $\widetilde{I}$. By Lemma 
3.6 we have $\beta(\widetilde{I}) \subseteq \widetilde{I}$ and $\beta_{*}(\widetilde{I}) \subseteq \widetilde{I}$. Hence we see that $\beta_{*}^{k}\left(q a_{k}\right)$ is in $\widetilde{I}$ if and only if $a_{k} \in I^{\prime}$. Similarly $\beta_{*}^{n}\left(a_{n}\right)$ is in $\widetilde{I}$ if and only if $a_{n} \in I$.

Accordingly, we have shown that (18) yields an injective map from the set of invariant ideals in $(B, \beta)$ to the set of $J$-pairs for $(A, \alpha)$. To prove it is surjective let $\left(I, I^{\prime}\right)$ be an arbitrary $J$-pair for $(A, \alpha)$ and view $(B, \beta)$ as the direct limit of (11). Put $I_{k}:=\bar{\alpha}^{k}(1) I \bar{\alpha}^{k}(1)$ and $I_{k}^{\prime}:=\bar{\alpha}^{k}(1) I^{\prime} \bar{\alpha}^{k}(1), k \in \mathbb{N}$. For each $n \in \mathbb{N}$, the formula

$$
\widetilde{I}_{n}:=q\left(I_{0}^{\prime}\right) \oplus q\left(I_{1}^{\prime}\right) \oplus \ldots \oplus q\left(I_{n-1}^{\prime}\right) \oplus I_{n}
$$

defines an ideal in $B_{n}$ (recall that $q: A \rightarrow A / J$ is the quotient map). Relations saying that $\left(I, I^{\prime}\right)$ is a $J$-pair imply that $\widetilde{I}_{n+1} \cap T_{n}\left(B_{n}\right)=T_{n}\left(\widetilde{I}_{n}\right), n \in \mathbb{N}$. Hence with the identifications from Remark 3.3, we deduce that the closure $\widetilde{I}$ of the ascending sum $\bigcup_{n \in \mathbb{N}} \widetilde{I}_{n}$ is an ideal in $B$ satisfying (18) and (19). We still need to show that $\widetilde{I}$ is invariant in $(B, \beta)$. Using description of $\beta_{*}$ given in the proof of Theorem 3.1 we get

$$
\beta_{*}\left(\widetilde{I}_{n}\right)=0 \oplus q\left(I_{1}^{\prime}\right) \oplus q\left(I_{2}^{\prime}\right) \oplus \ldots \oplus q\left(I_{n}^{\prime}\right) \oplus I_{n+1} \subseteq \widetilde{I}_{n+1} .
$$

Therefore $\beta_{*}(\widetilde{I}) \subseteq \widetilde{I}$. Similarly, we have $\beta(\widetilde{I}) \subseteq \widetilde{I}$ because

$$
\beta\left(\widetilde{I}_{n+1}\right)=q\left(I_{1}^{\prime}\right) \oplus q\left(I_{2}^{\prime}\right) \oplus \ldots \oplus q\left(I_{n}^{\prime}\right) \oplus I_{n+1} \subseteq \widetilde{I}_{n} .
$$

Consequently, $\widetilde{I}$ is invariant by Lemma 3.6. This shows the bijective correspondence between $J$-pairs in $(A, \alpha)$ and invariant ideals in $(B, \beta)$. Plainly, it preserves the order. In particular, by Lemma 3.6 both the set of invariant ideals in $(B, \beta)$ and the set of $J$-pairs in $(A, \alpha)$ are lattices.

Now fix an ideal $\widetilde{I}$ invariant in $(B, \beta)$ and let $\left(I, I^{\prime}\right)$ be the corresponding $J$-pair. Let $\left(B^{\widetilde{I}}, \beta^{\widetilde{I}}\right)$ be the direct limit of the direct sequence

$$
\left(B_{0}^{\widetilde{I}}, \beta_{0}^{\widetilde{I}}\right) \stackrel{T_{0}^{\widetilde{I}}}{\longrightarrow}\left(B_{1}^{\widetilde{I}}, \beta_{1}^{\widetilde{I}}\right) \stackrel{T_{1}^{\widetilde{I}}}{\longrightarrow}\left(B_{2}^{\widetilde{I}}, \beta_{2}^{\widetilde{I}}\right) \stackrel{T_{2}^{\tilde{I}}}{\longrightarrow} \ldots
$$

where

$$
\begin{gathered}
B_{n}^{\widetilde{I}}:=q_{I^{\prime}}\left(A_{0}\right) \oplus q_{I^{\prime}}\left(A_{1}\right) \oplus \ldots \oplus q_{I^{\prime}}\left(A_{n-1}\right) \oplus q_{I}\left(A_{n}\right), \\
\beta_{n}^{\widetilde{I}}\left(q_{I^{\prime}}\left(a_{0}\right) \oplus \ldots \oplus q_{I^{\prime}}\left(a_{n-1}\right) \oplus q_{I}\left(a_{n}\right)\right):=q_{I^{\prime}}\left(a_{1}\right) \oplus \ldots \oplus q_{I^{\prime}}\left(a_{n}\right) \oplus q_{I}\left(\alpha\left(a_{n}\right)\right)
\end{gathered}
$$

and

$$
T_{n}^{\widetilde{I}}\left(q_{I^{\prime}}\left(a_{0}\right) \oplus \ldots \oplus q_{I^{\prime}}\left(a_{n-1}\right) \oplus q_{I}\left(a_{n}\right)\right)=q_{I^{\prime}}\left(a_{0}\right) \oplus \ldots \oplus q_{I^{\prime}}\left(a_{n}\right) \oplus q_{I}\left(\alpha\left(a_{n}\right)\right)
$$

for $a_{k} \in A_{k}, k=0, \ldots, n$. Note that, as $\alpha(I) \subseteq I$ and $I \subseteq I^{\prime}$, the $C^{*}$-dynamical systems $\left(B_{n}^{\widetilde{I}}, \beta_{n}^{\widetilde{I}}\right)$ and their bonding maps $T_{n}^{\widetilde{I}}$ are well defined.

Since $I, J \subseteq I^{\prime}$ we have the natural isomorphisms

$$
(A / J) / q\left(I^{\prime}\right) \cong A / I^{\prime} \cong(A / I) / q_{I}\left(I^{\prime}\right)
$$

It is not hard to convince yourself that they induce natural equivalences

$$
\left(B / \widetilde{I}, \beta_{\widetilde{I}}\right) \cong\left(B^{\widetilde{I}}, \beta^{\widetilde{I}}\right) \cong\left(B_{\left(I, I^{\prime}\right)}, \beta_{\left(I, I^{\prime}\right)}\right)
$$

Indeed, let $\left(B_{0}^{\left(I, I^{\prime}\right)}, \beta_{0}^{\left(I, I^{\prime}\right)}\right) \stackrel{T_{0}^{\left(I, I^{\prime}\right)}}{\longrightarrow}\left(B_{1}^{\left(I, I^{\prime}\right)}, \beta_{1}^{\left(I, I^{\prime}\right)}\right) \stackrel{T_{1}^{\left(I, I^{\prime}\right)}}{\longrightarrow}\left(B_{2}^{\left(I, I^{\prime}\right)}, \beta_{2}^{\left(I, I^{\prime}\right)}\right) \stackrel{T_{2}^{\left(I, I^{\prime}\right)}}{\longrightarrow} \ldots$ be the direct sequence defining $\left(B_{\left(I, I^{\prime}\right)}, \beta_{\left(I, I^{\prime}\right)}\right)$ and retain the notation form the first part of the proof. Then the isomorphisms (20) extend (by direct sums and restrictions) to isomorphisms making the 
following diagram commutative

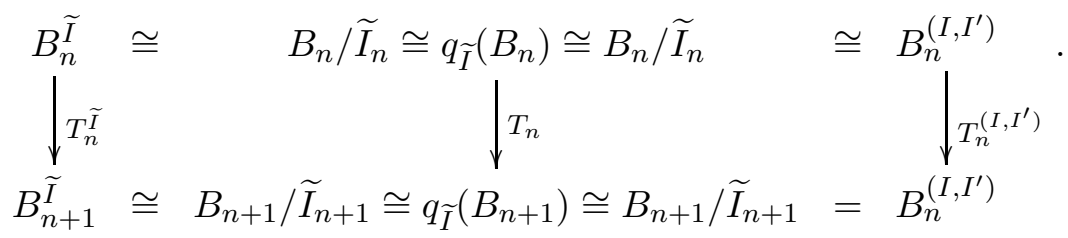

The horizontal isomorphisms establish equivalences $\left(B_{n}^{\widetilde{I}}, \beta_{n}^{\widetilde{I}}\right) \cong\left(B_{n} / \widetilde{I}_{n},\left(\beta_{n}\right)_{\widetilde{I}_{n}}\right) \cong\left(B_{1}^{\left(I, I^{\prime}\right)}, \beta_{1}^{\left(I, I^{\prime}\right)}\right)$ which imply equivalences of the limiting systems.

Corollary 3.13. Suppose $\operatorname{ker} \alpha$ is a complemented ideal in $A$ and let $(B, \beta)$ be a natural reversible extension of $(A, \alpha)$. We have an order preserving bijection between the invariant ideals in systems $(A, \alpha)$ and $(B, \beta)$. It is given by the relations

$$
I=\widetilde{I} \cap A, \quad \widetilde{I}=\sum_{n=0}^{\infty} \beta_{*}^{n}(I) .
$$

Moreover, the subsystem $\left(B / \widetilde{I}, \beta_{\widetilde{I}}\right)$ corresponding to $\widetilde{I}$ is equivalent to the natural reversible extension of the subsystem $\left(A / I, \alpha_{I}\right)$ corresponding to $I$.

Proof. Within the notation of Theorem 3.12 we necessarily have $I^{\prime}=I+(\operatorname{ker} \alpha)^{\perp}$ (see Remark 3.8) and $q=1-\bar{\beta}_{*}(1)$ projects $A$ onto ker $\alpha$. Therefore $q I^{\prime}=I \cap$ ker $\alpha \subseteq I$, and consequently formula (19) implies that $\widetilde{I}=\sum_{n=0}^{\infty} \beta_{*}^{n}(I)$. Since $q_{I}\left(I^{\prime}\right)=q_{I}\left((\operatorname{ker} \alpha)^{\perp}\right)=\left(\operatorname{ker} \alpha_{I}\right)^{\perp}$ the second part of the assertion follows from Theorem 3.12 .

Remark 3.14. For an arbitrary $C^{*}$-dynamical system $(A, \alpha)$ and an ideal $J$ in $(\operatorname{ker} \alpha)^{\perp}$ relations (21) establish an order preserving bijection between the $J$-invariant ideals in $(A, \alpha)$ and invariant ideals in $(B, \beta)$ that are generated by their intersection with $A$. Indeed, the latter ideals correspond via bijection from Theorem 3.12 to $J$-pairs of the form $(I, I+J)$ and as $q(I+J)=q I$ the formula (19) reduces to $\widetilde{I}=\sum_{n=0}^{\infty} \beta_{*}^{n}(I)$.

It is not an immediate fact that the set of $J$-invariant ideals in $(A, \alpha)$ is a lattice.

Corollary 3.15. For any $C^{*}$-dynamical system $(A, \alpha)$ and any ideal $J$ in $(\operatorname{ker} \alpha)^{\perp}$ the set of $J$-invariant ideals in $(A, \alpha)$ is a lattice.

Proof. Let $I_{1}$ and $I_{2}$ be $J$-invariant ideals. It is evident that the intersection $I_{1} \cap I_{2}$ is a $J$ invariant ideal, which yields a natural meet operation. For the existence of join operation note that $\left(I_{1}, I_{1}+J\right)$ and $\left(I_{2}, I_{2}+J\right)$ are $J$-pairs, and therefore by Theorem 3.12 there exists the join $J$-pair:

$$
\left(I, I^{\prime}\right)=\left(I_{1}, I_{1}+J\right) \vee\left(I_{2}, I_{2}+J\right) .
$$

Plainly, $I$ is a $J$-invariant ideal containing $I_{1}$ and $I_{2}$. Moreover, if $I_{1}, I_{2} \subseteq K$ for a certain $J$-invariant ideal $K$ then

$$
\left(I_{1}, I_{1}+J\right),\left(I_{2}, I_{2}+J\right) \subseteq(K, K+J) \Longrightarrow\left(I, I^{\prime}\right) \subseteq(K, K+J) \Longrightarrow I \subseteq K .
$$

That is, $I$ is the minimal $J$-invariant ideal containing $I_{1}, I_{2}$. Hence $J$-invariant ideals form a lattice.

Another consequence of Theorem 3.12 is that the universal property of natural reversible $J$-extensions described in item ii) of Theorem 3.1 can be extended to not necessarily injective morphisms. 
Corollary 3.16. Let $(B, \beta)$ be a natural reversible $J$-extension of $(A, \alpha)$. If $(A, \alpha) \stackrel{S}{\rightarrow}(C, \gamma)$ is a morphism covariant on $J$ and $(C, \gamma)$ is reversible then $S$ extends uniquely to a covariant morphism $(B, \beta) \stackrel{\widetilde{S}}{\rightarrow}(C, \gamma)$.

Proof. By Lemma 3.11, $I:=\operatorname{ker} S$ and $I^{\prime}:=S^{-1}\left((\operatorname{ker} \gamma)^{\perp}\right)$ form a $J$-pair for $(A, \alpha)$. Clearly, $S$ factors through to a $q\left(I^{\prime}\right)$-embedding $\left(A / I, \alpha_{I}\right) \stackrel{S_{I}}{\rightarrow}(C, \gamma)$. Hence by Theorem 3.1 , using the notation and equivalence from Theorem $3.12, S_{I}$ extends uniquely to the covariant embedding $\left(B / \widetilde{I}, \beta_{\widetilde{I}}\right) \cong\left(B_{\left(I, I^{\prime}\right)}, \beta_{\left(I, I^{\prime}\right)}\right) \stackrel{\widetilde{S}_{I}}{\rightarrow}(C, \gamma)$. Thus $\widetilde{S}=\widetilde{S}_{I} \circ q_{\widetilde{I}}$ defines the desired extended morphism. It is unique because the invariant ideal $\widetilde{I}=\operatorname{ker} \widetilde{S}$ is uniquely determined by $\left(I, I^{\prime}\right)$.

\section{Representations of $C^{*}$-Dynamical systems. Crossed products}

We adopt the point of view that crossed products are $C^{*}$-algebras encoding representation theory of $C^{*}$-dynamical systems. A fundamental remark for our analysis is that any representation of $(A, \alpha)$ can be viewed as a morphism to a reversible $C^{*}$-dynamical system. Thus the notion of covariance transfers naturally from morphisms to representations. This leads us to the introduction of relative crossed products $C^{*}(A, \alpha, J)$.

4.1. Covariant representations and morphisms to reversible systems. Representations of $(A, \alpha)$ are defined in the following straightforward manner, cf. [31, Definition 1.1].

Definition 4.1. A Representation of a $C^{*}$-dynamical system $(A, \alpha)$ on a Hilbert space $H$ is a pair $(\pi, U)$ where $\pi: A \rightarrow \mathcal{B}(H)$ is a non-degenerate representation and $U \in \mathcal{B}(H)$ is such that

$$
U \pi(a) U^{*}=\pi(\alpha(a)), \quad a \in A .
$$

If $\pi$ is injective we say $(\pi, U)$ is a faithful. We denote by $C^{*}(\pi, U)$ the $C^{*}$-algebra generated by $\pi(A) \cup \pi(A) U$ and refer to it as to the $C^{*}$-algebra generated by $(\pi, U)$.

Remark 4.2. One can show (see Proposition 4.9 below) that if $(\pi, U)$ is a representation of $(A, \alpha)$ then $\pi: A \rightarrow C^{*}(\pi, U)$ is a non-degenerate homomorphism and $U \in M\left(C^{*}(\pi, U)\right)$. Thus we could consider abstract representations $(\pi, U)$ of $(A, \alpha)$ where $\pi: A \rightarrow C$ is a nondegenerate homomorphism into a $C^{*}$-algebra $C$ and $U \in M(C)$ is such that (22) holds. But then composing $\pi$ with any non-degenerate representation of $C$ on $H$ we get back to Definition 4.1 .

It is a remarkable consequence of multiplicativity of $\alpha$ that any representation of $(A, \alpha)$ defines a morphism to a reversible $C^{*}$-dynamical system.

Proposition 4.3. Let $(\pi, U)$ be a representation of $(A, \alpha)$ in a $C^{*}$-algebra $C$. Then $U$ is a (power) partial isometry,

$$
B:=\sum_{n=0}^{\infty} U^{* n} \pi(A) U^{n}
$$

is a $C^{*}$-algebra and

$$
U B U^{*} \subseteq B, \quad U^{*} B U \subseteq B, \quad U^{*} U \in B^{\prime}
$$

where $B^{\prime}$ is the commutant of $B$. In particular, putting $\beta(\cdot):=U(\cdot) U^{*}$ we get that $(B, \beta)$ is a reversible $C^{*}$-dynamical system with the complete transfer operator given by $\beta_{*}(\cdot):=U^{*}(\cdot) U$.

Proof. Since $\pi: A \rightarrow \mathcal{B}(H)$ is non-degenerate it extends uniquely to the strictly continuous unital homomorphism $\bar{\pi}: M(A) \rightarrow \mathcal{B}(H)$. Moreover, non-degeneracy of $\pi$ and strict continuity of $\bar{\alpha}$ readily imply that $(\bar{\pi}, U)$ is a representation of $(M(A), \bar{\alpha})$. Since for each $n \in \mathbb{N}, \pi\left(\bar{\alpha}^{n}(1)\right)=$ $U^{* n} U^{n}$ is a projection, $U$ is a power partial isometry. By [31, Lemma 1.2] we have $U^{* n} U^{n} \in$ $\bar{\pi}(M(A))^{\prime}$ and hence all the more $U^{* n} U^{n} \in \pi(A)^{\prime}$. 
Let $\beta(\cdot):=U(\cdot) U^{*}$ and $\beta_{*}(\cdot):=U^{*}(\cdot) U$. For each $N \in \mathbb{N}$, the self-adjoint linear space $\sum_{n=0}^{N} \beta_{*}^{k}(\pi(A))$ is a $*$-algebra because for $l \leq k$ and $a, b \in A$, we have

$$
\begin{aligned}
\beta_{*}^{k}(\pi(a)) \beta_{*}^{l}(\pi(b)) & =U^{* k} \pi(a) \beta^{k}(1) U^{k-l} \beta^{l}(1) \pi(b) U^{l} \\
& =U^{* k} \pi\left(a \bar{\alpha}^{k}(1)\right) U^{k-l}\left(U^{*(k-l)} U^{k-l}\right) \pi\left(\bar{\alpha}^{k}(1) b\right) U^{l} \\
& =U^{* k} \pi\left(a \bar{\alpha}^{k}(1)\right) U^{k-l} \pi\left(\bar{\alpha}^{l}(1) b\right) U^{*(k-l)} U^{k} \\
& =\beta_{*}^{k}\left(\pi\left(a \bar{\alpha}^{k}(1) \alpha^{k-l}(b)\right)\right) \in \beta_{*}^{k}(\pi(A)) .
\end{aligned}
$$

Hence $B:=\sum_{k=0}^{\infty} \beta_{*}^{k}(\pi(A))$ is a $C^{*}$-algebra. Relations $U^{*} B U \subseteq B$ and $U^{*} U \in B^{\prime}$ are now straightforward. Moreover, for any $a \in A$ and $k>0$ the projection $U^{* k-1} U^{k-1}$ commute with $\bar{\pi}(\bar{\alpha}(1)) \in \bar{\pi}(M(A))$ and thus we have

$$
\begin{aligned}
\beta\left(\beta_{*}^{k}(\pi(a))\right) & =\bar{\pi}(\bar{\alpha}(1))\left(U^{* k-1} U^{k-1}\right) U^{* k-1} \pi(a) U^{* k-1}\left(U^{* k-1} U^{k-1}\right) \bar{\pi}(\bar{\alpha}(1)) \\
& =\beta_{*}^{k-1}\left(\pi\left(\bar{\alpha}^{k}(1) a \bar{\alpha}^{k}(1)\right)\right) .
\end{aligned}
$$

This implies that $U B U^{*} \subseteq B$. Using relations (23) it is easy to see that $(B, \beta)$ is a reversible $C^{*}$-dynamical system. Indeed, for $a, b \in B$ we have

$$
\begin{gathered}
\beta(a b)=U\left(U^{*} U\right) a b U^{*}=U a\left(U^{*} U\right) b U^{*}=\beta(a) \beta(b), \\
a \beta_{*}(b)=a\left(U^{*} U\right) U^{*} b U=\left(U^{*} U\right) a U^{*} b U=\beta_{*}(\beta(a) b)
\end{gathered}
$$

and $\beta\left(\beta_{*}(a)\right)=\beta(1) a \beta(1)$.

Remark 4.4. Let $(\pi, U)$ be a representation of $(A, \alpha)$ and let $(B, \beta)$ be the associated $C^{*}$ dynamical system defined in the assertion of Proposition 4.3. Treating $\pi$ as a morphism $(A, \alpha) \stackrel{\pi}{\rightarrow}(B, \beta)$ we have

$$
\left\{a \in A: U^{*} U \pi(a)=\pi(a)\right\}=\pi^{-1}\left((\operatorname{ker} \beta)^{\perp}\right),
$$

cf. Lemma 2.14. Thus if $J$ is an ideal in $(\operatorname{ker} \alpha)^{\perp}$ then the morphism $(A, \alpha) \stackrel{\pi}{\rightarrow}(B, \beta)$ is covariant on $J$ if and only if the representation $(\pi, U)$ is covariant on $J$ in the following sense.

Definition 4.5 (cf. [31, Definition 1.7). Let $(\pi, U)$ be a representation of $(A, \alpha)$ and let $J$ be an ideal in $(\operatorname{ker} \alpha)^{\perp}$. We say that $(\pi, U)$ is covariant on $J$ if

$$
J \subseteq\left\{a \in A: U^{*} U \pi(a)=\pi(a)\right\} .
$$

If the above inclusion is the equality we say $(\pi, U)$ is a $J$-covariant representation. If $(\pi, U)$ is covariant on $(\operatorname{ker} \alpha)^{\perp}$ we say $(\pi, U)$ is a covariant representation.

By Remark 4.4 we can immediately translate Lemmas 2.11, 2.12 and Proposition 2.13 from the language of morphisms to the language of representations. For example, from Proposition 2.13 we get

Proposition 4.6. Let $(A, \alpha)$ be a reversible system and $(\pi, U)$ its representation. The following conditions are equivalent:

i) $(\pi, U)$ is covariant,

ii) $U^{*} \pi(a) U=\pi\left(\alpha_{*}(a)\right)$ for all $a \in A$,

iii) $\bar{\pi}\left(\bar{\alpha}_{*}(1)\right) \leq U^{*} U$,

iv) $\bar{\pi}\left(\bar{\alpha}_{*}(1)\right)=U^{*} U$.

We also immediately conclude with the following statement, cf. [29, Theorem 3.7]. 
Proposition 4.7. Let $(B, \beta)$ be a natural reversible $J$-extension of a $C^{*}$-dynamical system $(A, \alpha)$ where $J$ is an ideal in $(\operatorname{ker} \alpha)^{\perp}$. We have a one-to-one correspondence between representations $(\pi, U)$ of $(A, \alpha)$ covariant on $J$ and covariant representations $(\tilde{\pi}, U)$ of $(B, \beta)$ where

$$
\pi=\left.\tilde{\pi}\right|_{A}, \quad \tilde{\pi}\left(\sum_{k=0}^{n} \beta_{*}\left(a_{k}\right)\right)=\sum_{k=0}^{n} U^{*} \pi\left(a_{k}\right) U, \quad a_{k} \in A, k=0, \ldots, n .
$$

Under this correspondence ker $\widetilde{\pi}$ is an invariant ideal in $(B, \beta)$ corresponding to the $J$-pair for $(A, \alpha)$ given by

$$
I=\operatorname{ker} \pi, \quad I^{\prime}=\left\{a \in A: U^{*} U \pi(a)=\pi(a)\right\} .
$$

In particular, $\tilde{\pi}$ is faithful if and only if $\pi$ is faithful and $J=\left\{a \in A: U^{*} U \pi(a)=\pi(a)\right\}$.

Proof. In view of Remark 4.4 it suffices to apply Corollary 3.16 and Theorem 3.12 ,

4.2. Crossed products. We define the relevant crossed products in universal terms. They can be treated as a subclass of crossed products we proposed in [25, Definition 4.9] or in [28, Definition 2.3]. If $A$ is unital they coincide with those constructed and investigated in [31.

Definition 4.8. Let $(A, \alpha)$ be a $C^{*}$-dynamical system and $J$ an ideal in $(\operatorname{ker} \alpha)^{\perp}$. A crossed product of $A$ by $\alpha$ relative to $J$ is the $C^{*}$-algebra $C^{*}(A, \alpha, J):=C^{*}(\iota, u)$ generated by a representation $(\iota, u)$ of $(A, \alpha)$ which is universal with respect to covariance on $J$; i.e. we require that if $(\pi, U)$ is a representation of $(A, \alpha)$ covariant on $J$ then

$$
\iota(a) \longmapsto \pi(a), \quad u \longmapsto U
$$

extends to the homomorphism $\pi \rtimes U$ from $C^{*}(A, \alpha, J)$ onto $C^{*}(\pi, U)$. If $J=(\operatorname{ker} \alpha)^{\perp}$ we write $C^{*}(A, \alpha):=C^{*}\left(A, \alpha,(\operatorname{ker} \alpha)^{\perp}\right)$ and call it the (unrelative) crossed product of $A$ by $\alpha$.

Proposition 4.9. For any $C^{*}$-dynamical system $(A, \alpha)$ and any ideal $J$ in $(\operatorname{ker} \alpha)^{\perp}$, the crossed product $C^{*}(A, \alpha, J)$ exists and is unique (up to natural isomorphism).

Moreover,

i) the universal representation $(\iota, u)$ is faithful, $J$-covariant, and we have

$$
C^{*}(A, \alpha, J)=\overline{\operatorname{span}}\left\{u^{* n} \iota(a) u^{m}: a \in A, n, m \in \mathbb{N}\right\}, \quad u \in M\left(C^{*}(A, \alpha, J)\right) .
$$

ii) $C^{*}(A, \alpha, J)$ sits naturally as an ideal in $C^{*}(M(A), \bar{\alpha}, \bar{J})$.

iii) If $(B, \beta)$ is a natural reversible $J$-extension of $(A, \alpha)$ we have natural isomorphism

$$
C^{*}(A, \alpha, J) \cong C^{*}(B, \beta),
$$

and $C^{*}(B, \beta)$ is the closure of $a^{*}$-algebra consisting of elements of the form

$$
b=\sum_{k=1}^{n} u^{* n} \iota\left(b_{-k}\right)+\iota\left(b_{0}\right)+\sum_{k=1}^{n} \iota\left(b_{k}\right) u^{n}, \quad b_{ \pm k} \in B, k=0, \pm 1, \ldots, \pm n,
$$

where $(\iota, u)$ denotes the universal covariant representation of $(B, \beta)$.

Proof. Uniqueness of $C^{*}(A, \alpha, J)$ follows from universality. The existence can be shown by standard arguments, see [8]. But we deduce it, along the way, from the constructions performed in [31] for unital $C^{*}$-algebras. Namely, by [31, Theorem 1.11, Proposition 3.7] we know that the crossed product $C^{*}(M(A), \bar{\alpha}, \bar{J})$ exists and is generated by a faithful $\bar{J}$-covariant representation $(\bar{\iota}, u)$ of $(M(A), \bar{\alpha})$ on a certain Hilbert space $H$. Let $\iota:=\left.\bar{\iota}\right|_{A}$. We claim that, modulo non-degeneracy issues to be explained below, the pair $(\iota, u)$ is the universal $J$-covariant representation of $(A, \alpha)$.

Indeed, by 31] we know that $C^{*}(M(A), \bar{\alpha}, \bar{J})=\overline{\operatorname{span}}\left\{u^{* n} \bar{\iota}(a) u^{m}: a \in M(A), n, m \in \mathbb{N}\right\}$. Consider the self-adjoint Banach space $C^{*}(A, \alpha, J):=\overline{\operatorname{span}}\left\{u^{* n} \bar{\iota}(a) u^{m}: a \in A, n, m \in \mathbb{N}\right\}$. Let 
$a, b \in M(A)$ and $k, l, m, n \in \mathbb{N}$, where for instance $m \leq l$. Calculations similar to that in the proof of Proposition 4.3 give

$$
\left(u^{* k} \bar{\iota}(a) u^{l}\right)\left(u^{* m} \bar{\iota}(b) u^{n}\right)=u^{* k} \bar{\iota}\left(a \bar{\alpha}^{l-m}\left(\bar{\alpha}^{m}(1) b\right)\right) u^{n+l-m} .
$$

Hence if either $a$ or $b$ is in $A$ the product is in $u^{* k} \iota(A) u^{n+l-m}$. This shows that $C^{*}(A, \alpha, J)$ is an ideal in $C^{*}(M(A), \bar{\alpha}, \bar{J})$. In particular, $C^{*}(A, \alpha, J)$ is a $C^{*}$-algebra generated by $\iota(A) \cup$ $\iota(A) u$, and the orthogonal projection $P$ from $H$ onto the essential space $C^{*}(A, \alpha, J) H$ for $C^{*}(A, \alpha, J)$ commutes with elements of $C^{*}(M(A), \bar{\alpha}, \bar{J})$. Significantly, $P$ commutes with $u$. Furthermore, since $u^{* k} \iota(A)=u^{* k} \bar{\iota}\left(\bar{\alpha}^{k}(1)\right) \iota(A)=u^{* k} \iota\left(\alpha^{k}(A) A\right)=\iota(A) u^{* k} \iota(A)$ we see that $C^{*}(A, \alpha, J)$ contains $\iota(A)$ as a non-degenerate $C^{*}$-algebra. Thus $\iota(A) H=P H$ and $\iota(a)=\iota(a) P$ for all $a \in A$. Accordingly, restricting $\iota$ and $u$ to $P H$ we see that $(\iota, u)$ is a faithful representation of $(A, \alpha)$ on $P H$. It is $J$-covariant because

$$
\left\{a \in A: u^{*} u \iota(a) P=\iota(a) P\right\}=\left\{a \in A: u^{*} u \iota(a)=\iota(a)\right\}=A \cap \bar{J}=J,
$$

cf. Lemma 1.1. Universality of $(\iota, u)$ follows from Lemma 2.11 i), cf. Remark 4.4, and the universality of $C^{*}(M(A), \bar{\alpha}, \bar{J})$. Operator $u$ restricted to $P H$ identifies with a multiplier of $C^{*}(A, \alpha, J)$ in an obvious way. This shows parts i) and ii).

Part iii) follows from Proposition 4.7 and part i) of the present assertion, plus a simple observation that for $b \in B$ we have

$$
u^{* m}(b) u^{n}= \begin{cases}u^{*(m-n)} \iota\left(\beta_{*}^{n}(b)\right), & \text { if } m \geq n, \\ \iota\left(\beta_{*}^{m}(b)\right) u^{n-m}, & \text { if } m<n .\end{cases}
$$

Remark 4.10. In the sequel, we will assume the identification

$$
C^{*}(A, \alpha, J)=C^{*}(B, \beta)
$$

where $(B, \beta)$ is the natural reversible $J$-extension of $(A, \alpha)$. Moreover, we will write $(\iota, u)$ for both the universal covariant representation of $(B, \alpha)$ and the universal $J$-covariant representation of $(A, \alpha)$.

Remark 4.11. One can define the crossed product $C^{*}(A, \alpha, J)$ for an arbitrary ideal $J$ in $A$. Just let $C^{*}(A, \alpha, J)=C^{*}(\iota(A), u)$ be the $C^{*}$-algebra generated by a representation $(\iota, u)$ of $(A, \alpha)$ universal with respect to representations satisfying (24). However, if $J \nsubseteq(\operatorname{ker} \alpha)^{\perp}$ the universal representation $(\iota, u)$ is not faithful. Moreover, by the reduction procedure described in [31, Section 5.3], see also [25, Example 6.24],

$$
R:=\left\{a \in A: \alpha^{n}(a) \in J \text { for all } n \in \mathbb{N} \text { and } \lim _{n \rightarrow \infty} \alpha^{n}(a)=0\right\}
$$

is the smallest positively invariant ideal in $(A, \alpha)$ such that $q_{R}(J) \subseteq\left(\operatorname{ker} \alpha_{R}\right)^{\perp}$, and we have a natural isomorphism

$$
C^{*}(A, \alpha, J) \cong C^{*}\left(A / R, \alpha_{R}, q_{R}(J)\right) .
$$

Thus this seemingly more general situation reduces easily to that of Definition 4.8. For the particular case of Stacey's crossed product $A \times{ }_{\alpha}^{1} \mathbb{N}$ we have

$$
A \times{ }_{\alpha}^{1} \mathbb{N}=C^{*}(A, \alpha, A) \cong C^{*}\left(A / R, \alpha_{R}\right) \quad \text { where } \quad R=\left\{a \in A: \lim _{n \rightarrow \infty} \alpha^{n}(a)=0\right\} .
$$

Here $\left(A / R, \alpha_{R}\right)$ can be regarded as the largest subsystem of $(A, \alpha)$ such that $\alpha_{R}$ is a monomorphism. 
Let now $(A, \alpha)$ and $J \subseteq(\operatorname{ker} \alpha)^{\perp}$ be fixed. By universality of the representation $(\iota, u)$ generating $C^{*}(A, \alpha, J)$ we have a (point-wise continuous) circle action $\mathbb{T}=\{z \in \mathbb{C}:|z|=1\} \ni$ $z \longmapsto \gamma_{z} \in \operatorname{Aut}\left(C^{*}(A, \alpha, J)\right)$ determined by

$$
\gamma_{z}(\iota(a))=\iota(a), \quad \gamma_{z}(u)=z u, \quad a \in A, z \in \mathbb{T} .
$$

We denote this action by $\gamma$ and refer to it as the gauge action on $C^{*}(A, \alpha, J)$. One infers from Proposition 4.9 that the algebra $\iota(B)$, where $(B, \beta)$ is the natural reversible $J$-extension of $(A, \alpha)$, is the fixed point algebra for the gauge action $\gamma$. Integration over the Haar measure $\mu$ on $\mathbb{T}$ gives a conditional expectation

$$
E(a)=\int_{\mathbb{T}} \gamma_{z}(a) d \mu, \quad a \in C^{*}(A, \alpha, J),
$$

from $C^{*}(A, \alpha, J)$ onto $\iota(B)$. We get the following version of a standard result often called gauge-invariant uniqueness theorem.

Proposition 4.12. Let $(\pi, U)$ be a faithful $J$-covariant representation. The following conditions are equivalent:

i) $\pi \rtimes U: C^{*}(A, \alpha, J) \rightarrow C^{*}(\pi, U)$ is an isomorphism,

ii) we have the equality $J=\left\{a \in A: U^{*} U \pi(a)=\pi(a)\right\}$ and there is a circle action $\gamma^{\pi}$ on $C^{*}(\pi, U)$ such that

$$
\gamma_{z}^{\pi}(\pi(a))=\pi(a), \quad \gamma_{z}^{\pi}(U)=z U \quad a \in A, z \in \mathbb{T},
$$

iii) we have the equality $J=\left\{a \in A: U^{*} U \pi(a)=\pi(a)\right\}$ and there is a conditional expectation from $C^{*}(\pi, U)$ onto $\sum_{n=0}^{\infty} U^{* n} \pi(A) U^{n}$, sending the spaces $U^{* n} \pi(A) U^{m}$ to zero when $m \neq n$.

Proof. Implications i) $\Rightarrow$ ii) $\Rightarrow$ iii) follow easily from the discussion above and the last part of Proposition 4.7. To show iii) $\Rightarrow$ i) assume that $E^{\pi}$ is a conditional expectation from $C^{*}(\pi, U)$ onto $\sum_{n=0}^{\infty} U^{* n} \pi(A) U^{n}$, as described above. By the last part of Proposition 4.7 $\left.\pi \rtimes U\right|_{B}=\widetilde{\pi} \circ \iota$ is a faithful representation of $B$. By part iii) of Proposition 4.9 it suffices to show that $\pi \rtimes U(b) \neq 0$ if $b$ is a non-zero element given by (25). But if $b \neq 0$ is given by (25), then $u^{* k} \iota\left(b_{-k}\right) \neq 0$ or $\iota\left(b_{k}\right) u^{k} \neq 0$ for some $k=0, \pm 1, \ldots, \pm n$. Fix such $k$. Since

$$
0<u^{* k} \iota\left(b_{-k}\right) \iota\left(b_{-k}\right)^{*} u^{k}+\iota\left(b_{k}\right) u^{k} u^{* k} \iota\left(b_{k^{*}}\right)=\iota\left(\beta_{*}^{k}\left(b_{-k} b_{-k}^{*}\right)+b_{k} \bar{\beta}_{*}^{k}(1) b_{k}^{*}\right) \in \iota(B)
$$

we get

$$
0<\tilde{\pi}\left(\iota\left(\beta_{*}^{k}\left(b_{-k} b_{-k}^{*}\right)+b_{k} \bar{\beta}_{*}^{k}(1) b_{k}^{*}\right)\right) \leq E^{\pi}\left(\pi \rtimes U\left(b b^{*}\right)\right) .
$$

This implies that $\pi \rtimes U(b) \neq 0$.

4.3. Gauge-invariant ideals. Representations $\pi \rtimes U$ of $C^{*}(A, \alpha, J)$ satisfying condition ii) in Proposition 4.12 are sometimes called gauge-invariant representations. Kernels of such representations are gauge-invariant ideals in $C^{*}(A, \alpha, J)$, i.e. ideals that are invariant under the gauge action on $C^{*}(A, \alpha, J)$. Clearly, such ideals form a lattice. We describe this lattice in two steps.

Proposition 4.13. Let $(B, \beta)$ be a reversible $C^{*}$-dynamical system. Relations

$$
\begin{gathered}
\widetilde{I}=\{b \in B: \iota(b) \in \mathcal{I}\}, \\
\mathcal{I}=\sum_{k=1}^{\infty} u^{* k} \iota(\widetilde{I})+\iota(\widetilde{I})+\sum_{k=1}^{\infty} \iota(\widetilde{I}) u^{k},
\end{gathered}
$$


establish an order isomorphism between the lattices of gauge-invariant ideals $\mathcal{I}$ in $C^{*}(B, \beta)$ and invariant ideals $\widetilde{I}$ in $(B, \beta)$. Moreover, under the above correspondence we have

$$
C^{*}(B, \beta) / \mathcal{I} \cong C^{*}\left(B / \widetilde{I}, \beta_{\widetilde{I}}\right) .
$$

Proof. Let $\widetilde{I}$ be an invariant ideal in $(B, \beta)$ and define $\mathcal{I}$ by (27). To see that $\mathcal{I}$ is an ideal in $C^{*}(B, \beta)$ recall that the projections $u^{* k} u^{k}, k \in \mathbb{N}$, commute with elements of $\iota(B)$, and $u^{*} \iota(b) u=\iota\left(\beta_{*}(b)\right), b \in B$, see Propositions 4.6, 2.6. Hence for $a, b \in B$ and $m \geq n$, we get

$$
\begin{aligned}
& \iota(a) u^{m} \cdot u^{* n} \iota(b)=\iota\left(a \beta^{m-n}\left(\bar{\beta}^{n}(1) b\right)\right) u^{m-n}, \\
& u^{* n} \iota(b) \cdot \iota(a) u^{m}=\iota\left(\beta_{*}^{n}(b a)\right) u^{m-n} .
\end{aligned}
$$

Thus (by invariance of $\widetilde{I}$ ) if either $a$ or $b$ is in $\widetilde{I}$ then both of the above products are in $\mathcal{I}$. By passing to adjoints, we arrive at a similar conclusion for $m<n$. Accordingly, in view of Proposition 4.9 iii), $\mathcal{I}$ is an ideal in $C^{*}(B, \beta)$. Clearly, $\mathcal{I}$ is gauge-invariant and (26) holds. To show (28) denote by $\left(\iota_{\widetilde{I}}, u_{\widetilde{I}}\right)$ the universal representation generating the crossed product $C^{*}\left(B / \widetilde{I}, \beta_{\widetilde{I}}\right)$ and note that $\left(\iota_{\widetilde{I}} \circ q_{\widetilde{I}}, u_{\widetilde{I}}\right)$ is a covariant representation of $(B, \beta)$. Therefore we have the epimorphism

$$
C^{*}(B, \beta) \stackrel{\Psi}{\longmapsto} C^{*}\left(B / \widetilde{I}, \beta_{\widetilde{I}}\right) \quad \text { where } \quad \Psi(\iota(b))=\iota_{\widetilde{I}}\left(q_{\widetilde{I}}(b)\right), \quad \Psi(u)=u_{\widetilde{I}} .
$$

Since $\mathcal{I} \subseteq$ ker $\Psi$ this mapping factors through to an epimorphism

$$
C^{*}(B, \beta) / \mathcal{I} \stackrel{\Psi_{\mathcal{I}}}{\longmapsto} C^{*}\left(B / \widetilde{I}, \beta_{\widetilde{I}}\right) \quad \text { where } \quad \Psi_{\mathcal{I}} \circ q_{\mathcal{I}}=\Psi .
$$

As $\mathcal{I}$ is gauge-invariant, the gauge action on $C^{*}(B, \beta)$ factors through to $C^{*}(B, \beta) / \mathcal{I}$. With this circle action on $C^{*}(B, \beta) / \mathcal{I}, \Psi_{\mathcal{I}}$ becomes a gauge-invariant epimorphism which is injective on the fixed point algebra $q_{\mathcal{I}}(\iota(B))$. Hence by the general gauge-invariant uniqueness theorem, cf., for instance, [10, Theorem 4.5.1], $\Psi_{\mathcal{I}}$ is is injective on $C^{*}(B, \beta) / \mathcal{I}$. This proves (28).

Let now $\mathcal{I}^{\prime}$ be an arbitrary ideal in $C^{*}(B, \beta)$ and put $\widetilde{I}:=\left\{b \in B: \iota(b) \in \mathcal{I}^{\prime}\right\}$. Then $\iota(\beta(\widetilde{I}))=u \iota(\widetilde{I}) u^{*} \subseteq \mathcal{I}^{\prime}$ and $\iota\left(\beta_{*}(\widetilde{I})\right)=u^{*} \iota(\widetilde{I}) u \subseteq \mathcal{I}^{\prime}$. Thus $\widetilde{I}$ is invariant in $(B, \beta)$ by Lemma 3.6. Plainly, the gauge-invariant ideal $\mathcal{I}$ given by (27) is contained in $\mathcal{I}^{\prime}$. Accordingly, the identity factors through to the epimorphism

$$
C^{*}(B, \beta) / \mathcal{I} \stackrel{\Phi}{\longmapsto} C^{*}(B, \beta) / \mathcal{I}^{\prime} .
$$

If $\mathcal{I}^{\prime}$ is gauge-invariant then both of the algebras $C^{*}(B, \beta) / \mathcal{I}$ and $C^{*}(B, \beta) / \mathcal{I}^{\prime}$ are equipped with circle actions induced from $C^{*}(B, \beta)$. Epimorphism $\Phi$ is gauge-invariant with respect to these actions and $\Phi$ is injective on the fixed point algebra of the circle action on $C^{*}(B, \beta) / \mathcal{I}$. Hence $\Phi$ is an isomorphism, again by [10, Theorem 4.5.1]. Consequently, $\mathcal{I}=\mathcal{I}^{\prime}$.

Remark 4.14. The last part of the proof above shows that relation (26) establishes an order preserving surjection from the lattice of all ideals in $C^{*}(B, \beta)$ onto the lattice of invariant ideals in $(B, \beta)$. Thus the lattice of gauge-invariant ideals in $C^{*}(B, \beta)$ can be regarded as an order-retract of the lattice of all ideals.

Combining the above statement with Theorem 3.12 we obtain a general result.

Theorem 4.15. Let $(A, \alpha)$ be a $C^{*}$-dynamical system and $J$ an ideal in $(\operatorname{ker} \alpha)^{\perp}$. We have an order isomorphism from the lattice of gauge-invariant ideals $\mathcal{I}$ in $C^{*}(A, \alpha, J)$ onto the lattice of $J$-pairs $\left(I, I^{\prime}\right)$ for $(A, \alpha)$. It is determined by relations

$$
I=\{a \in A: \iota(a) \in \mathcal{I}\}, \quad I^{\prime}=\left\{a \in A:\left(1-u^{*} u\right) \iota(a) \in \mathcal{I}\right\},
$$

$\mathcal{I}$ is generated by $\iota(I)+\left(1-u^{*} u\right) \iota\left(I^{\prime}\right)$. 
For ideals satisfying the above relations we have

$$
C^{*}(A, \alpha, J) / \mathcal{I} \cong C^{*}\left(A / I, \alpha_{I}, q_{I}\left(I^{\prime}\right)\right) .
$$

Restricting the above order isomorphism we get an order preserving bijective correspondence between ideals $\mathcal{I}$ in $C^{*}(A, \alpha, J)$ which are generated by their intersection with $\iota(A)$, and $J$ invariant ideals $I$ in $(A, \alpha)$. This restricted correspondence is determined by relations

$$
I=\{a \in A: \iota(a) \in \mathcal{I}\}, \quad \mathcal{I}=\overline{\operatorname{span}}\left\{u^{* m} \iota(a) u^{n}: a \in I, n, m \in \mathbb{N}\right\},
$$

and for the corresponding ideals we have

$$
C^{*}(A, \alpha, J) / \mathcal{I} \cong C^{*}\left(A / I, \alpha_{I}, q_{I}(J)\right) .
$$

Proof. We obtain the desired order isomorphism by composing the isomorphisms from Proposition 4.13 and Theorem 3.12. Moreover, using (twice) the isomorphism from Proposition 4.9 iii), the equivalence of $C^{*}$-dynamical systems from Theorem 3.12 and the isomorphism (28) we get

$$
\begin{aligned}
C^{*}(A, \alpha, J) / \mathcal{I} & \cong C^{*}(B, \beta) / \mathcal{I} \cong C^{*}\left(B / \widetilde{I}, \beta_{\widetilde{I}}\right) \cong C^{*}\left(B_{\left(I, I^{\prime}\right)}, \beta_{\left(I, I^{\prime}\right)}\right) \\
& \cong C^{*}\left(A / I, \alpha_{I}, q_{I}\left(I^{\prime}\right)\right) .
\end{aligned}
$$

This proves the first part of the assertion.

For the second part note that the ideal $\mathcal{I}$ in $C^{*}(A, \alpha, J)$ generated by $I:=\mathcal{I} \cap \iota(A)$ corresponds, via the above isomorphism, to the $J$-pair $(I, I+J)$, see also Remark 3.14 .

Corollary 4.16. Suppose $\operatorname{ker} \alpha$ is a complemented ideal in A. Relations (30) establish an order isomorphism between the lattices of gauge-invariant ideals $\mathcal{I}$ in $C^{*}(A, \alpha)$ and invariant ideals $\widetilde{I}$ in $(A, \alpha)$. Under this correspondence we have

$$
C^{*}(A, \alpha) / \mathcal{I} \cong C^{*}\left(A / I, \alpha_{I}\right) .
$$

In particular, in this case every gauge-invariant ideal $\mathcal{I}$ in $C^{*}(A, \alpha)$ is generated by its intersection with $\iota(A)$.

Proof. Apply the fact that any $(\operatorname{ker} \alpha)^{\perp}$-pair in $(A, \alpha)$ is of the form $\left(I, I+(\operatorname{ker} \alpha)^{\perp}\right)$ where $I$ is invariant in $(A, \alpha)$, cf. Remark 3.8, In particular, we have $q_{I}\left((\operatorname{ker} \alpha)^{\perp}\right)=\left(\operatorname{ker} \alpha_{I}\right)^{\perp}$.

4.4. Simplicity. Now we use the above result to study simplicity of $C^{*}(A, \alpha, J)$. We will juxtapose the conditions we get with [46, Theorem 4.1], [42, Corollary 1] which provide certain sufficient conditions for simplicity of $C^{*}(A, \alpha)$ when $\alpha$ is a monomorphism. To deal with noninjective $\alpha$ we will use Corollary 4.28 from the next subsection, and a notion of pointwise quasinilpotence, which seems to be a novelty in the present context.

Definition 4.17. We say that $\alpha$ is minimal provided that there are no nontrivial invariant ideals in $(A, \alpha)$. We call $\alpha$ pointwise quasinilpotent if $\lim _{n \rightarrow \infty} \alpha^{n}(a)=0$, for all $a \in A$. A monomorphism $\alpha$ is called inner if there is an isometry $v \in M(A)$ such that $\alpha(a)=v a v^{*}$, $a \in A$.

Note that if $\alpha$ has no positively invariant ideals then $\alpha$ is necessarily a minimal monomorphism. Moreover, if $\alpha$ is a monomorphism and $A$ is unital, it can be shown that $\alpha$ is minimal if and only if there are no positively invariant ideals in $(A, \alpha)$, cf. [46], [40].

Theorem 4.18. If the algebra $C^{*}(A, \alpha, J)$ is simple then $J=(\operatorname{ker} \alpha)^{\perp}, \alpha$ is minimal and either $\alpha$ is pointwise quasinilpotent or $\alpha$ is a monomorphism and no power $\alpha^{n}, n>0$, is inner.

If $\alpha$ is minimal each of the following conditions imply that $C^{*}(A, \alpha)$ is simple:

i) $\alpha$ is pointwise quasinilpotent,

ii) $\alpha$ is injective, $A$ is unital, and no power $\alpha^{n}, n>0$, is inner,

iii) $\alpha$ is injective with hereditary range, $A$ is separable, and no power $\alpha^{n}, n>0$, is inner. 
Proof. As $(\{0\}, J)$ and $\left(\{0\},(\operatorname{ker} \alpha)^{\perp}\right)$ are always $J$-pairs, Theorem4.15implies that $C^{*}(A, \alpha, J)$ is not simple unless $J=(\operatorname{ker} \alpha)^{\perp}$. Similarly, $C^{*}(A, \alpha)$ is not simple unless there are no invariant ideals in $(A, \alpha)$. Moreover, one readily sees that

$$
I:=\left\{a \in A: \lim _{n \rightarrow \infty} \alpha^{n}(a)=0\right\}
$$

is an invariant ideal in $(A, \alpha)$. Thus if $C^{*}(A, \alpha)$ is simple then $\alpha$ is minimal and either $I=A$ or $I=\{0\}$, that is either $\alpha$ is pointwise quasinilpotent or $\alpha$ is a monomorphism.

Suppose then that $\alpha$ is a monomorphism and for some $n>0, \alpha^{n}$ is inner so that $\alpha^{n}(a)=$ $v a v^{*}$, for all $a \in A$, where $v \in M(A)$ is an isometry. We claim that we may reduce our considerations to the case $(A, \alpha)$ is a reversible $C^{*}$-dynamical system. Indeed, if $(B, \beta)$ is the natural reversible extension of $(A, \alpha)$ then the natural reversible extension of $\left(A, \alpha^{n}\right)$ can be identified with $\left(B, \beta^{n}\right)$. But $(i d, v)$ is a faithful covariant representation of $\left(A, \alpha^{n}\right)$ in $A$, cf. Remark 4.2. Thus by Proposition 4.7 we have the isomorphism $B \cong \sum_{k=0}^{\infty} v^{* k} A v^{k}=A$ under which $\beta^{n}(b)=v b v^{*}, b \in B$, and $v \in M(B)$. This proves our claim. Let us then assume that $(A, \alpha)$ is reversible. In view of Proposition 4.6 we have $\bar{\alpha}_{*}^{n}(a)=v^{*} a v$ for all $a \in M(A)$. In particular $\bar{\alpha}_{*}^{n}(v)=v^{*} v v=v$. For each $m=1, \ldots, n$ we put $v_{m}:=\bar{\alpha}_{*}^{m}(v)$. Note that for $a \in A$ we have

$$
v_{m} a=\bar{\alpha}_{*}^{m}(v) a=\bar{\alpha}_{*}^{m}\left(v \alpha^{m}(a)\right)=\bar{\alpha}_{*}^{m}\left(\alpha^{m+n}(a) v\right)=\alpha^{n}(a) \bar{\alpha}_{*}^{m}(v)=\alpha^{n}(a) v_{m} .
$$

Since $\bar{\alpha}^{n}(1) v=v$, all the more $\bar{\alpha}^{m}(1) v=v$ and thus we get

$$
v_{m}^{*} v_{m}=\bar{\alpha}_{*}^{m}\left(v^{*} \bar{\alpha}^{m}\left(\bar{\alpha}_{*}^{m}(v)\right)\right)=\bar{\alpha}_{*}^{m}\left(v^{*} \bar{\alpha}^{m}(1) v \bar{\alpha}^{m}(1)\right)=\bar{\alpha}_{*}^{m}\left(v^{*} v\right)=\bar{\alpha}_{*}^{m}(1)=1 .
$$

Thus $v_{m} \in M(A)$ are isometries such that $\alpha^{n}(a)=v_{m} a v_{m}^{*}$ for all $a \in A$. These isometries commute. Indeed, $\bar{\alpha}^{n}\left(v_{k}\right)=v_{k} v_{k} v_{k}^{*}=v_{k} \bar{\alpha}^{n}(1)=v_{k} v_{m} v_{m}^{*}$ and $\bar{\alpha}^{n}\left(v_{k}\right)=v_{m} v_{k} v_{m}^{*}$ imply that $v_{k} v_{m}=v_{k} v_{m}$, for $m, k=1, \ldots, n$. Moreover, for $m=1, \ldots, n$ (and $v_{0}=v$ ) we have $\bar{\alpha}\left(v_{m}\right)=$ $\bar{\alpha}(1) v_{m-1} \bar{\alpha}(1)=v_{m-1} \bar{\alpha}(1)$. Thus putting

$$
w:=v_{0} v_{1} \ldots v_{n-1}
$$

we get an isometry in $M(A)$ such that

$$
w a w^{*}=\alpha^{n^{2}}(a) \quad a \in A, \quad \text { and } \quad \bar{\alpha}(w)=w \bar{\alpha}(1) .
$$

Now assume that $A$ is faithfully represented as a $C^{*}$-subalgebra of $\mathcal{B}(H)$ acting non-degenerately on $H$. Put $\widetilde{H}:=H \oplus \bar{\alpha}(1) H \oplus \ldots \oplus \bar{\alpha}^{n^{2}-1}(1) H$. Using (31) one readily checks that the formulae

$$
\pi(a):=\operatorname{diag}\left(a, \alpha(a), \ldots, \alpha^{n^{2}-1}(a)\right), \quad U=\left(\begin{array}{ccccc}
0 & \bar{\alpha}(1) & 0 & \ldots & 0 \\
0 & 0 & \bar{\alpha}^{2}(1) & \ldots & 0 \\
\vdots & \vdots & \vdots & \ddots & \vdots \\
0 & 0 & 0 & \ldots & \bar{\alpha}^{n^{2}-1}(1) \\
w & 0 & 0 & \ldots & 0
\end{array}\right)
$$

define a covariant representation $(\pi, U)$ of $(A, \alpha)$ on $\widetilde{H}$. Moreover, $U^{n^{2}}=\bar{\pi}(w)$ where $\bar{\pi}$ is the extension of $\pi$ onto $M(A)$. Hence $(\pi, U)$ gives rise to a representation $\pi \rtimes U$ of $C^{*}(A, \alpha)$ which is faithful on $\iota(A)$ but not globally faithful because for any $a \in A$ we have

$$
(\pi \rtimes U)\left(\iota(w a)-u^{n^{2}} \iota(a)\right)=0 .
$$

This contradicts the simplicity of $C^{*}(A, \alpha)$.

Part i) follows from Theorem 4.15 and Corollary 4.28 (which we prove in next section). Part ii) follows from [46, Theorem 4.1], and part iii) follows from [42, Corollary 1] (note that the proof of [42, Theorem 1] is based on results of [41] which were proved under assumption that $A$ is separable and this assumption seems to be essential). 
4.5. Topological freeness for reversible $C^{*}$-dynamical systems. Throughout this subsection $(B, \beta)$ stands for a reversible $C^{*}$-dynamical system. One can think of $(B, \beta)$ as of the natural $J$-reversible extension of a certain (not necessarily reversible) $C^{*}$-dynamical system $(A, \alpha)$. We will adopt this viewpoint in Corollary 4.28 below.

Let $\widehat{B}$ be the spectrum of $B$ equipped with the Jacobson topology. As it is generally accepted, we will abuse notation and use $\pi$ to denote both an irreducible representation of $B$ and its equivalence class in $\widehat{B}$. Since $\beta_{*}(B)=(\operatorname{ker} \beta)^{\perp}$ is an ideal in $B$ and $\beta(B)=\bar{\beta}(1) B \bar{\beta}(1)$ is a hereditary subalgebra of $B$ we have natural identifications of spectra of $\beta_{*}(B)$ and $\beta(B)$ with open subsets of $\widehat{B}$ :

$$
\widehat{\beta_{*}(B)}=\left\{\pi \in \widehat{B}: \pi\left(\beta_{*}(B)\right) \neq 0\right\}, \quad \widehat{\beta(B)}=\{\pi \in \widehat{B}: \pi(\beta(B)) \neq 0\} .
$$

With these identifications the homeomorphisms dual to the mutually inverse isomorphisms $\beta: \beta_{*}(B) \rightarrow \beta(B)$ and $\beta_{*}: \beta(B) \rightarrow \beta_{*}(B)$ yield partial homeomorphisms of $\widehat{B}$ :

$$
\widehat{\beta}: \widehat{\beta(B)} \rightarrow \widehat{\beta_{*}(B)}, \quad \widehat{\beta}^{-1}=\widehat{\beta}_{*}: \widehat{\beta_{*}(B)} \rightarrow \widehat{\beta(B)},
$$

cf. also [31, 27]. More precisely, let $\pi: B \rightarrow \mathcal{B}(H)$ be a representation. If $\pi \in \widehat{\beta_{*}(B)}$ then $\widehat{\beta}_{*}(\pi)$ is the unique (up to unitary equivalence) irreducible extension of $\pi \circ \beta_{*}: \beta(B) \rightarrow \mathcal{B}(H)$ up to irreducible representation $\widehat{\beta}_{*}(\pi): B \rightarrow \mathcal{B}(\widetilde{H})$ where $\widehat{\beta}_{*}(\pi)(\beta(B)) \widetilde{H}=H \subseteq \widetilde{H}$. If $\pi \in \widehat{\beta(B)}$, then $\widehat{\beta}(\pi)$ is the unique extension of $\pi \circ \beta: \beta_{*}(B) \rightarrow \mathcal{B}(\beta(B) H)$ up to the (irreducible) representation $\widehat{\beta}(\pi): B \rightarrow \mathcal{B}(\beta(B) H)$.

Definition 4.19. We say that a partial homeomorphism $\varphi$ of a topological space $X$ is topologically free if the set of its periodic points of any given period $n>0$ has empty interior.

The following statements could be deduced from the results of [26], in the same fashion as [27, Theorem. 2.19]. However, we can omit the use of Hilbert bimodules by reducing the problem to the unital case treated in [27, Theorem 2.19], cf. also [31, Theorem 3.1].

Theorem 4.20. Let $(B, \beta)$ be a reversible $C^{*}$-dynamical system. If the dual partial homeomorphism $\widehat{\beta}$ is topologically free then for every faithful covariant representation $(\pi, U)$ of $(B, \beta)$, $\pi \rtimes U: C^{*}(B, \beta) \rightarrow C^{*}(\pi, U)$ is an isomorphism.

Proof. The extended system $(M(B), \bar{\beta})$ is reversible, see Proposition 2.6. With natural identifications $\widehat{B}$ is an open and dense subset of $\widehat{M(B)}$ and $\widehat{\beta}$ is restriction of the partial homeomorphism $\widehat{\bar{\beta}}$ of $\widehat{M(B)}$ dual to $\bar{\beta}$. Thus topological freeness of $\bar{\beta}$ is equivalent to topological freeness of $\widehat{\bar{\beta}}$. Moreover, by Lemma 2.11 iii), cf. Remark 4.4. $(\bar{\pi}, U)$ is a faithful covariant representation of $(M(B), \bar{\beta})$. Hence by [27, Theorem $2.19 \mathrm{i})], \bar{\pi} \rtimes U: C^{*}(M(B), \bar{\beta}) \rightarrow C^{*}(\bar{\pi}, U)$ is an isomorphism which (with the identification from Proposition 4.9 ii)) restricts to the isomorphism $\pi \rtimes U: C^{*}(B, \beta) \rightarrow C^{*}(\pi, U)$.

Remark 4.21. There are reasons to believe that topological freeness and the isomorphism property described in the assertion of Theorem 4.20 are equivalent, at least for a large class of $C^{*}$-algebras. For instance, by [41, Theorem 10.4] this is the case when $\beta$ is an automorphism and $B$ is separable. We adapt a standard construction to show that this is true when $B$ is commutative.

Example 4.22 (Orbit representation). Let $B$ be commutative. By an orbit in $(\widehat{B}, \widehat{\beta})$ we mean a maximal set $\mathcal{O} \subseteq \widehat{B}$ whose elements can be indexed by $\mathbb{Z}, \mathbb{Z}_{+}, \mathbb{Z}_{-}$, or $\{1 \ldots, n\}$, for $n>0$, in such a way that $\widehat{\beta}\left(x_{k}\right)=x_{k+1}$, if $x_{k}, x_{k+1} \in \mathcal{O}$, and $\widehat{\beta}\left(x_{n}\right)=x_{1}$ in the case $\mathcal{O}$ is a periodic orbit with minimal period $n$. To each $\mathcal{O}$ we attach a covariant representation $\left(\pi_{\mathcal{O}}, U_{\mathcal{O}}\right)$ of $(B, \beta)$ on 
B. K. KWAŚNIEWSKI

the Hilbert space $\ell^{2}(\mathcal{O})$ with orthonormal basis $\delta_{x_{k}}, x_{k} \in \mathcal{O}$. We let $\pi_{\mathcal{O}}$ to be the diagonal representation given by $\pi_{\mathcal{O}}(a) \delta_{x_{k}}=x_{k}(a) \delta_{x_{k}}, x_{k} \in \mathcal{O}$. We define $U$ as the shift

$$
U_{\mathcal{O}} \delta_{x_{k}}=\delta_{x_{k-1}} \text { if } x_{k-1} \in \mathcal{O}, \quad \text { and } \quad U_{\mathcal{O}} \delta_{x_{k}}=0 \text { if } x_{k-1} \notin \mathcal{O},
$$

unless $\mathcal{O}=\left\{x_{1}, \ldots, x_{n}\right\}$ is periodic and $k=1$ in which case we put $U_{\mathcal{O}} \delta_{x_{1}}=x_{n}$. The direct sums

$$
\pi:=\bigoplus \pi_{\mathcal{O}}, \quad U:=\bigoplus U_{\mathcal{O}}
$$

over all orbits in $(\widehat{B}, \widehat{\beta})$ define a faithful covariant representation of $(B, \beta)$. Suppose now that $\widehat{\beta}$ is not topologically free. Hence there is a nonempty open set $U$ consisting of periodic points of period $n$. Then for any non-zero element $b \in \bigcap_{x \in \widehat{B} \backslash U} \operatorname{ker} x$ we have

$$
0 \neq b-b u^{n} \in \operatorname{ker}(\pi \rtimes U) .
$$

Hence $\pi \rtimes U$ is not faithful. In particular, $\operatorname{ker}(\pi \rtimes U)$ is not gauge-invariant, cf. Proposition 4.12 .

Since invariant ideals in $(B, \beta)$ correspond to open sets invariant under $\widehat{\beta}$, Theorem 4.20 leads us to a condition implying that all ideals in $C^{*}(B, \beta)$ are gauge-invariant.

Definition 4.23. Let $\Delta$ be a domain of a partial homeomorphism $\varphi$ of $X$. A set $V \subseteq X$ is $\varphi$-invariant if $\varphi(V \cap \Delta)=V \cap \varphi(\Delta)$. We say that $\varphi$ is (residually) free, if it is topologically free when restricted to any closed invariant set.

Corollary 4.24. If $\widehat{\beta}$ is free then all ideals in $C^{*}(B, \beta)$ are gauge-invariant and thus we have natural isomorphisms between the lattices of the following objects:

i) ideals in $C^{*}(B, \beta)$,

ii) invariant ideals in $(B, \beta)$,

iii) open $\widehat{\beta}$-invariant subsets of $\beta$.

Moreover, if $B$ is commutative then $\widehat{\beta}$ is free if and only if all ideals in $C^{*}(B, \beta)$ are gaugeinvariant.

Proof. Let $\mathcal{I}^{\prime}$ be an ideal in $C^{*}(B, \beta)$ and denote by $\mathcal{I}$ the ideal in $C^{*}(B, \beta)$ generated by $\iota(B) \cap \mathcal{I}^{\prime}$. Recalling the last part of the proof of Proposition 4.13, we have the epimorphism

$$
C^{*}\left(B / \widetilde{I}, \beta_{\widetilde{I}}\right) \cong C^{*}(B, \beta) / \mathcal{I} \stackrel{\Phi}{\longmapsto} C^{*}(B, \beta) / \mathcal{I}^{\prime}
$$

which is injective on the image of $B / \widetilde{I}$. As $\widetilde{I}$ is invariant, the spectrum of $B / \widetilde{I}$ is identified with the $\widehat{\beta}$-invariant closed set $\widehat{B} \backslash \widehat{\widetilde{I}}$ and then $\widehat{\beta}_{\widetilde{I}}$ becomes a restriction of $\widehat{\beta}$. Thus if $\widehat{\beta}$ is free then $\left(\widehat{B / \widetilde{I}}, \widehat{\beta_{\widetilde{I}}}\right)$ is topologically free. Therefore, by Theorem 4.20, $\Phi$ is an isomorphism and consequently $\mathcal{I}^{\prime}=\mathcal{I}$ is gauge-invariant.

Assume that $B$ is commutative and $\widehat{\beta}$ is not free. Then there is an invariant ideal $\widetilde{I}$ in $(B, \beta)$ such that the partial homeomorphism $\widehat{\beta_{\widetilde{I}}}$ is not topologically free. By Example 4.22 there is an ideal $\mathcal{I}^{\prime}$ in $C^{*}\left(B / \widetilde{I}, \beta_{\widetilde{I}}\right)$, which is not gauge-invariant. Denoting by $\mathcal{I}$ the (gauge-invariant) ideal in $C^{*}(B, \beta)$ generated by $\iota(\widetilde{I})$ and identifying $C^{*}\left(B / \widetilde{I}, \beta_{\widetilde{I}}\right)$ with $C^{*}(B, \beta) / \mathcal{I}$ we get an ideal $q_{\mathcal{I}}^{-1}\left(\mathcal{I}^{\prime}\right)$ in $C^{*}(B, \beta)$ which fails to be gauge-invariant.

Corollary 4.25. If $\beta$ is a minimal monomorphism with hereditary range and $\widehat{\beta}$ is topologically free, then $C^{*}(B, \beta)$ is simple.

Remark 4.26. If $\beta$ is injective topological freeness of $\widehat{\beta}$ implies that no power $\alpha^{n}, n>0$, is inner. Hence Corollary 4.25 is consistent with Theorem 4.18 ii), see also Remark 4.21.

We finish this subsection with an application to general $C^{*}$-dynamical systems. 
Lemma 4.27. Let $(A, \alpha)$ be a $C^{*}$-dynamical system, $J$ an ideal in $(\operatorname{ker} \alpha)^{\perp}$ and $(B, \beta)$ the natural reversible $J$-extension of $(A, \alpha)$. Then $\alpha$ is pointwise quasinilpotent if and only if $\beta$ is pointwise quasinilpotent.

Proof. With the notation from the proof of Theorem 3.1, $\beta$ is determined by the formulas $\beta\left(\phi_{0}\left(a_{0}\right)\right)=\phi_{0}\left(\alpha\left(a_{0}\right)\right)$ and

$$
\left.\beta\left(\phi_{n}\left(q\left(a_{0}\right) \oplus \ldots \oplus q\left(a_{n-1}\right) \oplus a_{n}\right)\right)=\phi_{n-1}\left(q\left(a_{1}\right)\right) \oplus \ldots \oplus q\left(a_{n-1}\right) \oplus a_{n}\right),
$$

for $n>0, a_{k} \in A_{k}, k=0, \ldots, n$. Thus $\beta$ is pointwise quasinilpotent on $\bigcup_{n \in \mathbb{N}} \phi_{n}\left(B_{n}\right)$ if and only if $\alpha$ is pointwise quasinilpotent on $A$. The assertion follows by the density of $\bigcup_{n \in \mathbb{N}} \phi_{n}\left(B_{n}\right)$ in $B$.

Corollary 4.28. Let $(A, \alpha)$ be a $C^{*}$-dynamical system and $J$ an ideal in $(\operatorname{ker} \alpha)^{\perp}$. If $\alpha$ is pointwise quasinilpotent then all ideals in $C^{*}(A, \alpha, J)$ are gauge-invariant.

Proof. In view of Corollary 4.24 and Lemma 4.27 it suffices to note that if a reversible $C^{*}$ dynamical system $(B, \beta)$ is such that $\beta$ is pointwise quasinilpotent, then $\widehat{\beta}$ is free. But if $\widehat{\beta}$ is not free then $\widehat{\beta_{\widetilde{I}}}$, for a certain subsystem $\left(B / \widetilde{I}, \beta_{\widetilde{I}}\right)$ of $(B, \beta)$, is not topologically free.

This implies that there exists $b \in B / \widetilde{I}$, a representation $\pi \in \widehat{B / \widetilde{I}}$ and a number $n$ such that $0 \neq\|\pi(b)\|=\left\|\pi\left(\beta_{\widetilde{I}}^{n k}(b)\right)\right\|$ for all $k \in \mathbb{N}$. Hence $\beta$ can not be pointwise quasinilpotent.

4.6. Commutative $C^{*}$-dynamical systems. We fix a locally Hausdorff space $X$ and a $C^{*}$ dynamical system $(A, \alpha)$ where $A=C_{0}(X)$ is the algebra of continuous functions on $X$ that vanish at infinity. We also fix an ideal $J \subseteq(\operatorname{ker} \alpha)^{\perp}$ and denote by $(B, \beta)$ the natural reversible $J$-extension of $(A, \alpha)$. Clearly, $B$ is also commutative. We will identify $B$ with $C_{0}(\tilde{X})$ for some locally compact Hausdorff space $\widetilde{X}$. Then, for $a \in A$ and $b \in B$, we have

$$
\alpha(a)(x)=\left\{\begin{array}{ll}
a(\varphi(x)), & x \in \Delta, \\
0, & x \notin \Delta,
\end{array} \quad \beta(b)(x)= \begin{cases}b(\widetilde{\varphi}(\widetilde{x})), & \widetilde{x} \in \widetilde{\Delta}, \\
0, & \widetilde{x} \notin \widetilde{\Delta},\end{cases}\right.
$$

where $\varphi: \Delta \rightarrow X$ and $\widetilde{\varphi}: \widetilde{\Delta} \rightarrow \widetilde{X}$ are proper continuous mappings defined respectively on clopen subsets $\Delta \subseteq X$ and $\widetilde{\Delta} \subseteq \widetilde{X}$ (they are closed because $\alpha$ and $\beta$ are extendible). One deduces from Propositions $2.3,2.6$ that $\widetilde{\varphi}$ is a homeomorphism onto a clopen set $\widetilde{\varphi}(\widetilde{\Delta}) \subseteq \tilde{X}$ and $\beta_{*}$ is actually an extendible endomorphism given by the similar formula as $\beta$ but with $\tilde{\varphi}$ replaced with $\widetilde{\varphi}^{-1}$.

We dualize our construction of $(B, \beta)$ to obtain a description of $(\widetilde{X}, \widetilde{\varphi})$ in terms of $(X, \varphi)$. This end let $Y \subseteq X$ be the hull of the ideal $J$, that is $Y$ is the closed set such that

$$
J=C_{0}(X \backslash Y) \text {. }
$$

Note that the relation $J \subseteq(\operatorname{ker} \alpha)^{\perp}$ is equivalent to the equality $Y \cup \varphi(\Delta)=X$. In the unital case the following description, using a different approach, was obtained in [24, Theorem 3.5] and under an additional assumption in [30, Theorem 3.5].

Proposition 4.29. Up to a homeomorphism $\widetilde{X}$ is the following subspace of the product space $\prod_{n \in \mathbb{N}}(X \cup\{0\})$, where $\{0\}$ is an abstract clopen singleton:

$$
\widetilde{X}=\bigcup_{N=0}^{\infty} X_{N} \cup X_{\infty}
$$

where

$$
X_{N}=\left\{\left(x_{0}, x_{1}, \ldots, x_{N}, 0, \ldots\right): x_{n} \in \Delta, \varphi\left(x_{n}\right)=x_{n-1}, n=1, \ldots, N, x_{N} \in Y\right\}
$$

and

$$
X_{\infty}=\left\{\left(x_{0}, x_{1}, \ldots\right): x_{n} \in \Delta, \varphi\left(x_{n}\right)=x_{n-1}, n \geqslant 1\right\}
$$


Then $\widetilde{\Delta}=\left\{\left(x_{0}, x_{1}, \ldots\right) \in \widetilde{X}: x_{0} \in \Delta\right\}, \widetilde{\varphi}(\widetilde{\Delta})=\left\{\left(x_{0}, x_{1}, \ldots\right) \in \widetilde{X}: x_{1} \neq 0\right\}$, and

$$
\widetilde{\varphi}\left(x_{0}, x_{1}, \ldots\right)=\left(\varphi\left(x_{0}\right), x_{0}, x_{1}, \ldots\right), \quad \widetilde{\varphi}^{-1}\left(x_{0}, x_{1}, \ldots\right)=\left(x_{1}, \ldots\right) .
$$

Proof. Let $\Delta_{n}:=\varphi^{-n}(\Delta)$ be the natural domain of the partial mapping $\varphi^{n}$. Dualizing diagram (10) we see that direct sequence (11) dualizes to the following inverse sequences

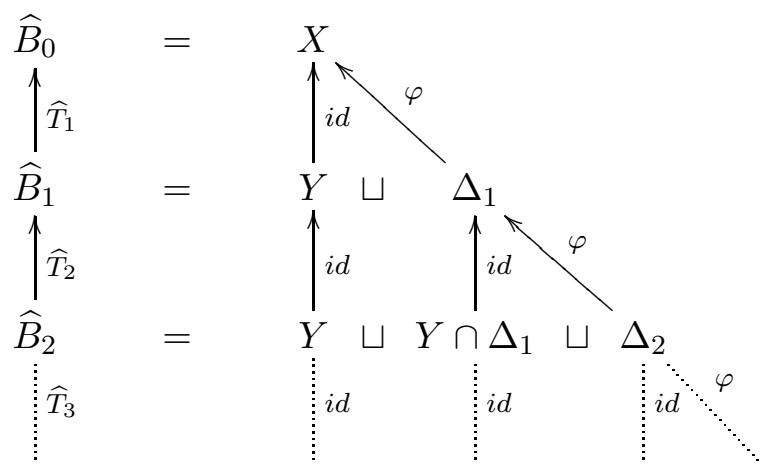

where $\sqcup$ denotes the direct sum of topological spaces. Now it is easy to see that the inverse limit $\varliminf_{\lim }\left\{\widehat{B}_{n}, \widehat{T}_{n}\right\}$ can be identified with the space $\widetilde{X}$ described in the assertion. For instance, if $\widetilde{x}=\left(x_{0}, x_{1}, x_{2}, \ldots\right)$ is an element of the inverse limit, then we have two alternatives: either every coordinate of $\widetilde{x}$ 'lies on the diagonal' of the above diagram, and then $\widetilde{x}$ identifies as the element of $X_{\infty}$; or only $N$ first coordinates of $\widetilde{x}$ 'lie on the diagonal' and next ones 'slide off the diagonal' fixing at $x_{N}$ - then we identify $\widetilde{x}$ with the element of $X_{N}$ by replacing the tail of $x_{N}$ 's with tail of zeros. We leave the remaining tedious details to the reader.

We extend Definition 4.19 to not (necessarily) injective maps.

Definition 4.30. We say that a periodic orbit $\mathcal{O}=\left\{x, \varphi(x), \ldots, \varphi^{n}(x)\right\}$ of a periodic point $x$ has an entrance $y \in \Delta$ if $y \notin \mathcal{O}$ and $\varphi(y) \in \mathcal{O}$. The partial mapping $\varphi$ is topologically free if the set of all periodic points whose orbits have no entrances has empty interior.

We will also need a relative version of topological freeness which coincides with the unrelative one when $Y=\overline{X \backslash \varphi(\Delta)}$ is the smallest set under consideration (recall that we must have $Y \cup \varphi(\Delta)=X)$.

Definition 4.31. The partial mapping $\varphi$ is said to be topologically free outside $Y$ if the set of periodic points whose orbits do not intersect $Y$ and have no entrances have empty interior.

Lemma 4.32. Let $(X, \varphi)$ and $(\widetilde{X}, \widetilde{\varphi})$ be as in Proposition 4.29. The partial homeomorphism $\widetilde{\varphi}$ is topologically free if and only if $\varphi$ is topologically free outside $Y$.

Proof. Let $n>0$. By Baire theorem it suffices to show that the set $\widetilde{F}_{n}:=\left\{\widetilde{x}: \widetilde{\varphi}^{n}(\widetilde{x})=\widetilde{x}\right\}$ has empty interior in $\widetilde{X}$ if and only if the set

$$
F_{n}:=\left\{x: \varphi^{n}(x)=x \text { and } \varphi^{-1}\left(\varphi^{k}(x)\right)=\left\{\varphi^{k-1}(x)\right\} \subseteq X \backslash Y, \text { for all } k=1, \ldots, n\right\}
$$

has empty interior in $X$. But if $U$ is a non-empty open subset of $F_{n}$ then our description of $(\widetilde{X}, \widetilde{\varphi})$ implies that $\widetilde{U}:=\left\{x \in \widetilde{X}: x_{0} \in U\right\}$ is non-empty open subset of $\widetilde{F}_{n}$. Conversely, if $\widetilde{F}_{n}$ has a non-empty interior then $\widetilde{F}_{n}$ contains a non-empty set of the form $\widetilde{U}:=\left\{x \in \widetilde{X}: x_{n} \in U\right\}$ for an open set $U$ in $X$. The inclusion $\widetilde{U} \subseteq \widetilde{F}_{n}$ forces $U$ to be contained in $F_{n}$.

Combining the above lemma and Theorem 4.20, modulo the last part of Proposition 4.7 and Example 4.22, we get

Proposition 4.33. The following conditions are equivalent: 
i) the partial map $\varphi$ is topologically free outside $Y$ (the hull of the ideal $J$ ),

ii) any faithful representation $(\pi, U)$ of $(A, \alpha)$ such that $J=\left\{a \in A: U^{*} U \pi(a)=\pi(a)\right\}$ give rises to a faithful representation $C^{*}(A, \alpha, J)$

Remark 4.34. If $Y=X$ then any $\varphi$ is (trivially) topologically free outside $Y$. Hence regardless of $(A, \alpha)$ any faithful representation $(\pi, U)$ of $(A, \alpha)$ such that $\left\{a \in A: U^{*} U \pi(a)=\pi(a)\right\}=\{0\}$, integrates to the isomorphism from $C^{*}(A, \alpha,\{0\})$ onto $C^{*}(\pi, U)$. This is a resemblance of much more general facts whose prototype is Coburn's uniqueness theorem.

We define objects dual to $J$-pairs and $J$-invariant ideals as follows

Definition 4.35. A set $V \subseteq X$ is positively invariant under $\varphi$ if $\varphi(V \cap \Delta) \subseteq V$, and $V$ is $Y$-negatively invariant if $V \subseteq Y \cup \varphi(V \cap \Delta)$. If $V$ is both positively and $Y$-negatively invariant, we call it $Y$-invariant. A pair $\left(V, V^{\prime}\right)$ of closed subsets of $X$ such that

$$
V \text { is positively } \varphi \text {-invariant, } \quad V^{\prime} \subseteq Y \text { and } \quad V^{\prime} \cup \varphi(V \cap \Delta)=V
$$

will be called a $Y$-pair for $(X, \varphi)$. We equip the set $Y$-pairs with natural partial order: $\left(V_{1}, V_{1}^{\prime}\right) \subseteq$ $\left(V_{2}, V_{2}^{\prime}\right) \stackrel{\text { def }}{\Longleftrightarrow} V_{1} \subseteq V_{2}$ and $V_{1}^{\prime} \subseteq V_{2}^{\prime}$.

Proposition 4.36. All ideals in $C^{*}(A, \alpha, J)$ are gauge-invariant if and only if $\varphi$ has no periodic points. In general, the relations

$$
C_{0}(X \backslash V)=\{a \in A: \iota(a) \in \mathcal{I}\}, \quad C_{0}\left(X \backslash V^{\prime}\right)=\left\{a \in A:\left(1-u^{*} u\right) \iota(a) \in \mathcal{I}\right\},
$$

establish an anti-isomorphism between the lattices of gauge-invariant ideals $\mathcal{I}$ in $C^{*}(A, \alpha, J)$ and $Y$ pairs $\left(V, V^{\prime}\right)$ for $(X, \varphi)$; it restricts to the anti-isomorphism between the lattices of all ideals $\mathcal{I}$ in $C^{*}(A, \alpha, J)$ generated by their intersection with $\iota(A)$ and $Y$-invariant closed sets $V$ for $(X, \varphi)$.

Proof. Since $\widetilde{X}$ is Hausdorff, freeness of $\widetilde{\varphi}$ (Definition 4.23) is equivalent to nonexistence of periodic points for $\widetilde{\varphi}$. In view of our description of $\widetilde{\varphi}$ the latter is equivalent to nonexistence of periodic points for $\varphi$. Thus the initial part of the assertion follows from Corollary 4.24. The second part follows from Theorem 4.15 modulo a simple remark that a pair $\left(C_{0}(X \backslash V), C_{0}(X \backslash\right.$ $\left.\left.V^{\prime}\right)\right)$ forms a $J$-pair for $(A, \alpha)$ if and only if $\left(V, V^{\prime}\right)$ form as a $Y$-pair for $(X, \varphi)$.

We say that a (full) mapping $\varphi: X \rightarrow X$ is minimal provided there are no nontrivial closed subsets $V$ of $X$ such that $\varphi(V)=V$. If $X$ is compact then $\varphi$ is minimal if and only if there are no nontrivial closed positively invariant subsets $V$ of $X$, i.e. such that $\varphi(V) \subseteq V$. However, when $X$ is not compact the latter condition is much stronger then minimality. In fact, by [17, Theorem B], nonexistence of nontrivial closed positively invariant subsets in $(X, \varphi)$ forces $X$ to be compact. On the other hand, there are interesting minimal mappings on non-compact spaces, cf. for instance [14].

Theorem 4.37. Let $(A, \alpha)$ be a commutative $C^{*}$-dynamical system and $(X, \varphi)$ its dual partial dynamical system. The crossed product $C^{*}(A, \alpha)$ is simple if and only one of the two possible cases hold:

i) $X$ is discrete, $\varphi$ is injective and $X$ compose of one non-periodic orbit $\mathcal{O}$. In this case $C^{*}(A, \alpha) \cong \mathcal{K}\left(\ell^{2}(\mathcal{O})\right)$ is the algebra of compact operators on the $|\mathcal{O}|$-dimensional Hilbert space.

ii) $X$ is not discrete and $\varphi: X \rightarrow X$ is a minimal surjection. In this case $C^{*}(A, \alpha) \cong$ $C^{*}(\widetilde{X}, \widetilde{\varphi})$ is the $C^{*}$-algebra of the minimal homeomorphism $\widetilde{\varphi}$ induced by $\varphi$ on the inverse limit space $\widetilde{X}$. 
B. K. KWAŚNIEWSKI

Proof. Let $C^{*}(A, \alpha)$ be simple. Assume that $\varphi: \Delta \rightarrow \varphi(\Delta)$ is a homeomorphism and $\varphi(\Delta) \subseteq X$ is clopen (think of it as of the system $(\widetilde{X}, \widetilde{\varphi})$ described in Proposition 4.29). Evidently, any orbit $\mathcal{O}$ and hence its closure is a $\varphi$-invariant set, cf. Definition 4.23. Therefore, by Corollary 4.24, $X$ is the closure of $\mathcal{O}$ and $\mathcal{O}$ is not a periodic orbit.

Suppose that $X$ is discrete. Then $X=\mathcal{O}$. Since $\mathcal{O}$ is not periodic, $(X, \varphi)$ is up to conjugacy either a truncated shift on $\{1, \ldots, n\}$, one sided shift on $\mathbb{N}$, or a two-sided shift on $\mathbb{Z}$. In each of these cases representations described in Example 4.22 yield the isomorphism $C^{*}(A, \alpha) \cong$ $\mathcal{K}\left(\ell^{2}(\mathcal{O})\right)$, cf. Theorem 4.20 .

Now let $X$ be arbitrary. We claim that if $\Delta \neq X$ then $X$ is discrete. Indeed, if $X \backslash \Delta=$ $\overline{\mathcal{O}} \backslash \Delta \neq \emptyset$ then $\mathcal{O} \backslash \Delta=\left\{x_{0}\right\}$ must be a singletone. As $\mathcal{O} \subseteq \Delta \cup\left\{x_{0}\right\}$ we actually have $X=\overline{\mathcal{O}}=\Delta \cup\left\{x_{0}\right\}$. Therefore $\left\{x_{0}\right\}$ is clopen in $X$. Consequently, $\mathcal{O}=\bigcup_{n \in \mathbb{N}} \varphi^{-n}\left(x_{0}\right)$ is an open discrete set. Clearly it is $\varphi$-invariant, and thus by minimality $X=\mathcal{O}=\bigcup_{n \in \mathbb{N}} \varphi^{-n}\left(x_{0}\right)$ is discrete.

Applying the above argument to the system $\left(X, \varphi^{-1}\right)$ we see that $X$ is discrete also when $\varphi(\Delta) \neq X$. Thus if $X$ is not discrete then $\Delta=\varphi(\Delta)=X$ and this finishes the proof in the reversible case.

Now suppose $(X, \varphi)$ is a general partial dynamical system (with $\varphi$ not necessarily injective). Then as we have shown the reversible system $(\widetilde{X}, \widetilde{\varphi})$ described in Proposition 4.29 satisfies the assertion. A moment of thought leads to the conclusion that if $(\widetilde{X}, \widetilde{\varphi})$ is as described in the case i) then $(X, \varphi)=(\widetilde{X}, \widetilde{\varphi})$. Similarly, $\widetilde{\varphi}: \widetilde{X} \rightarrow \widetilde{X}$ is a full minimal homeomorphism iff $\varphi: X \rightarrow X$ is a full minimal surjection.

\section{Appendix: $C^{*}(A, \alpha, J)$ viewed as a Relative Cuntz-Pimsner Algebra}

In this section we briefly outline how to obtain our description of the lattice of gauge-invariant ideals using general machinery of relative Cuntz-Pimsner algebras. For more details, concerning the latter we refer the reader to [38], 31], [19].

A $C^{*}$-correspondence over a $C^{*}$-algebra $A$ is a right Hilbert $A$-module $X$ with a left action $\phi_{X}: A \rightarrow(X)$ of $A$ on $X$ via adjointable operators. We let $J(X):=\phi^{-1}(\mathcal{K}(X))$ to be the ideal in $A$ consisting of elements that act from the left on $X$ as generalized compact operators. For any ideal $J$ in $J(X)$ the relative Cuntz-Pimsner algebra $\mathcal{O}(J, X)$ is constructed as a quotient of the $C^{*}$-algebra generated by Fock representation of $X$, see [38, Definition 2.18] or [31, Definition 4.9]. The $C^{*}$-algebra $\mathcal{O}(J, X)$ is universal with respect to appropriately defined representations of $X$, see [16, Remark 1.4] or [31, Proposition 4.10]. It is equipped with a gauge circle action which acts as identity on the image of $A$ in $\mathcal{O}(J, X)$. Katsura, in [21], described ideals in $\mathcal{O}(J, X)$ that are invariant under this action in the following way.

For any ideal $I$ in $A$ we define two another ideals

$$
\begin{gathered}
X(I):=\overline{\operatorname{span}}\left\{\langle x, a \cdot y\rangle_{A} \in A: a \in I, x, y \in X\right\}, \\
X^{-1}(I):=\left\{a \in A:\langle x, a \cdot y\rangle_{A} \in I \text { for all } x, y \in X\right\} .
\end{gathered}
$$

If $X(I) \subseteq I$, then the ideal $I$ is said to be positively invariant, [21, Definition 4.8]. For any positively invariant ideal $I$ we have a naturally defined quotient $C^{*}$-correspondence $X_{I}=X / X I$ over $A / I$. Denoting by $q_{I}: A \rightarrow A / I$ the quotient map one puts

$$
J_{X}(I):=\left\{a \in A: \phi_{X_{I}}\left(q_{I}(a)\right) \in \mathcal{K}\left(X_{I}\right), a X^{-1}(I) \subseteq I\right\} .
$$

Definition 5.1 (Definition 5.6 in [21]). Let $X$ be a $C^{*}$-correspondence over a $C^{*}$-algebra $A$. A $T$-pair of $X$ is a pair $\left(I, I^{\prime}\right)$ of ideals $I, I^{\prime}$ of $A$ such that $I$ is positively invariant and $I \subseteq I^{\prime} \subseteq J_{X}(I)$.

The content of [21, Proposition 11.9] is the following: 
Theorem 5.2. Let $X$ be a $C^{*}$-correspondence over a $C^{*}$-algebra $A$, and $J$ be an ideal of $A$ contained in $J(X)$. Then there exists a one-to-one correspondence between the set of all gaugeinvariant ideals of $O(J, X)$ and the set of all T-pairs $\left(I, I^{\prime}\right)$ of $X$ satisfying $J \subseteq I^{\prime}$, which preserves inclusions and intersections.

Let us now fix a $C^{*}$-dynamical system $(A, \alpha)$ and an ideal $J \subseteq(\operatorname{ker} \alpha)^{\perp}$. We associate to $(A, \alpha)$ a $C^{*}$-correspondence $X_{\alpha}$ which as a vector space is equal to $\bar{\alpha}(1) A$ and the other operations are given by

$$
a \cdot x:=\alpha(a) x \quad x \cdot a:=x a, \quad\langle x, y\rangle_{A}:=x^{*} y, \quad a \in A, x, y \in X_{\alpha} .
$$

One can show that $J\left(X_{\alpha}\right)=A$. Hence we can consider the relative Cuntz-Pimsner algebra $\mathcal{O}\left(J, X_{\alpha}\right)$. Moreover, there is a natural isomorphism

$$
C^{*}(A, \alpha, J) \cong \mathcal{O}\left(J, X_{\alpha}\right)
$$

which maps $\iota(A)$ and $u^{*} \iota(A)$, respectively, onto the image of $A$ and $X_{\alpha}$ in $\mathcal{O}\left(J, X_{\alpha}\right)$. The latter fact was noticed in [16, Example 1.6] and [31, Corollary 4.14] under the assumption that $A$ is unital, but the arguments carry out easily to our more general situation. In particular, (32) induces a lattice isomorphism between the gauge-invariant ideals in $C^{*}(A, \alpha, J)$ and $\mathcal{O}\left(J, X_{\alpha}\right)$.

Clearly, for any ideal $I$ in $A$ we have $X_{\alpha}(I)=A \alpha(I) A$ and $X_{\alpha}^{-1}(I)=\alpha^{-1}(I)$. In particular, $I$ is positively invariant for $X$ if and only if $I$ is positively invariant in $(A, \alpha)$. If $I$ is positively invariant then the quotient $C^{*}$-correspondence $X / X I$ can be identified with the $C^{*}$ correspondence $X_{\alpha_{I}}$ associated to the quotient system $\left(A / I, \alpha_{I}\right)$. Hence $J\left(X_{I}\right)=A / I$ and we get $J_{X_{\alpha}}(I)=\left\{a \in A: a \alpha^{-1}(I) \subseteq I\right\}$. Accordingly, if $I$ and $I^{\prime}$ are ideals in $A$ we have

$$
\left(I, I^{\prime}\right) \text { is a } T \text {-pair with } J \subseteq I^{\prime} \Longleftrightarrow\left(I, I^{\prime}\right) \text { is a } J \text {-pair for }(A, \alpha) \text {. }
$$

In this way we can infer from Theorem 5.2 the first part of Theorem 4.15 .

Corollary 5.3. Let $(A, \alpha)$ be a $C^{*}$-dynamical system and $J$ an ideal in $(\operatorname{ker} \alpha)^{\perp}$. We have an order isomorphism from the lattice of gauge-invariant ideals $\mathcal{I}$ in $C^{*}(A, \alpha, J)$ onto the lattice of $J$-pairs $\left(I, I^{\prime}\right)$ for $(A, \alpha)$.

In a similar way, using isomorphism (32), one can apply to $C^{*}(A, \alpha, J)$ other general results for relative Cuntz-Pimsner algebras. For instance, it follows from [20, Theorem 7.1] and [20, Theorem 7.2], respectively that

$$
A \text { is exact } \Longleftrightarrow C^{*}(A, \alpha, J) \text { is exact. }
$$$$
A \text { is nuclear } \Longrightarrow C^{*}(A, \alpha, J) \text { is nuclear. }
$$

If $A$ is separable, then $C^{*}(A, \alpha, J)$ is separable and by the argument leading to 20, Proposition 8.8] we have

$$
\text { both } A \text { and } J \text { satisfy the UCT } \Longrightarrow C^{*}(A, \alpha, J) \text { satisfy the UCT. }
$$

\section{REFERENCES}

[1] S. Adji, Crossed products of $C^{*}$-algebras by semigroups of endomorphisms, Ph.D. thesis, University of Newcastle, 1995.

[2] S. Adji, M. Laca, M. Nilsen, I. Raeburn, Crossed products by semigroups of endomorphisms and the Toeplitz algebras of ordered groups, Proc. Amer. Math. Soc. 122 (1994), 1133-1141.

[3] A. B. Antonevich, V. I. Bakhtin, A. V. Lebedev, Crossed product of a $C^{*}$-algebra by an endomorphism, coefficient algebras and transfer operators, Math. Sbor. 202(9) (2011), 1253-1283.

[4] A. B. Antonevich, A. V. Lebedev, Functional differential equations: I. $C^{*}$-theory, Longman Scientific \& Technical, Harlow, Essex, England, 1994.

[5] P. Ara, M. A. González-Barroso, K. R. Goodearl, E. Pardo, Fractional skew monoid rings, J. Algebra 278 (2004), 104-126.

[6] J. Arledge, M. Laca, I. Raeburn, Semigroup crossed products and Hecke algebras arising from number fields, Doc. Math. 2 (1997), 115-138. 
[7] T. Bates, J. H. Hong, I. Raeburn, W. Szymański, The ideal structure of the $C^{*}$-algebras of infinite graphs, Illinois J. Math. 46(4) (2002), 1159-1176.

[8] B. Blackadar, Shape theory for $C^{*}$-algebras, Math. Scand. 56 (1985), 249-275.

[9] S. Boyd, N. Keswani, I. Raeburn, Faithful representations of crossed products by endomorphisms, Proc. Amer. Math. Soc. 118 (1993), 427-436.

[10] N. Brown, N. Ozawa, $C^{*}$-algebras and Finite-Dimensional Approximations, Grad. Stud. Math. 88, Amer. Math. Soc. Providence, RI 2008.

[11] N. Brownlowe, I. Raebrun, S. T. Vittadello, Exel's crossed product for non-unital $C^{*}$-algebras, Math. Proc. Cambridge Phil. Soc. 149 (2010), 423-444.

[12] J. Cuntz, Simple $C^{*}$-algebras generated by isometries, Commun. Math. Phys. 57 (1977), 173-185.

[13] J. Cuntz, The internal structure of simple $C^{*}$-algebras, Proc. Sympos. Pure Math. 38 (1982), 85-115.

[14] A. I. Danilenko, Strong orbit equivalence of locally compact Cantor minimal systems, Internat. J. Math. 12(1) (2001), 113-123.

[15] R. Exel, A new look at the crossed-product of a $C^{*}$-algebra by an endomorphism, Ergodic Theory Dynam. Systems 23 (2003), 1733-1750.

[16] N. J. Fowler, P. S, Muhly, I. Raeburn, Representations of Cuntz-Pimsner algebras, Indiana Univ. Math. J. 52 (2003) 569-605.

[17] W. H. Gottschalk, Orbit-closure decompositions and almost periodic properties, Bull. Amer. Math. Soc. 50 (1944), 915-919.

[18] A. an Huef, I. Raeburn, Stacey crossed products associated to Exel systems, Integr. Equ. Oper. Theory 72 (2012), 537-561.

[19] T. Katsura, A construction of $C^{*}$-algebras from $C^{*}$-correspondences, Contemp. Math, 335, Amer. Math. Soc., Providence, RI, 2003, 173-182.

[20] T. Katsura, On $C^{*}$-algebras associated with $C^{*}$-correspondences, J. Funct. Anal., 217 (2004), No 2, 366-401

[21] T. Katsura, Ideal structure of $C^{*}$-algebras associated with $C^{*}$-correspondences, Pacific J. Math. 230 (2007), 107-145.

[22] A. Kishimoto, Outer automorphisms and reduced crossed products of simple $C^{*}$-algebras, Comm. Math. Phys. 81 (1981), 429-435.

[23] B. K. Kwaśniewski, On transfer operators for $C^{*}$-dynamical systems, Rocky J. Math. 42(3) (2012), 919-938.

[24] B. K. Kwaśniewski, $C^{*}$-algebras associated with reversible extensions of logistic maps, Mat. Sb. 203(10) (2012), 1448-1489

[25] B. K. Kwaśniewski, $C^{*}$-algebras generalizing both relative Cuntz-Pimsner and Doplicher-Roberts algebras, Trans. Amer. Math. Soc. 365 (2013), 1809-1873.

[26] B.K. Kwaśniewski, Topological freeness for Hilbert bimodules, Israel J. Math. 199(2) (2014), 641-650

[27] B. K. Kwaśniewski, Crossed products by interactions and graph algebras, Integr. Equ. Oper. Theory 80(3) (2014), 415-451

[28] B. K. Kwaśniewski, Exel's crossed products and crossed products by completely positive maps, preprint arXiv:1404.4929

[29] B. K. Kwaśniewski, Extensions of $C^{*}$-dynamical systems to systems with complete transfer operators, preprint arXiv:math/0703800

[30] B. K. Kwaśniewski, A.V. Lebedev, Reversible extensions of irreversible dynamical systems: $C^{*}$-method, Math. Sbor. 199(11) (2008), 45-74.

[31] B. K. Kwaśniewski, A.V. Lebedev, Crossed products by endomorphisms and reduction of relations in relative Cuntz-Pimsner algebras, J. Funct. Anal. 264(8) (2013), 1806-1847.

[32] M. Laca, Semigroups of *-endomorphisms, Dirichlet series and phase transitions, J. Funct. Anal. 152 (1998), 330-378.

[33] M. Laca, From endomorphisms to automorphisms and back: dilations and full corners, J. London Math. Soc. 61(2) (2000), 893-904.

[34] M. Laca, I. Raeburn, Semigroup crossed products and the Toeplitz algebras of nonabelian groups, J. Funct. Anal., 139 (1996), 415-440.

[35] M. Laca, I. Raeburn, A semigroup crossed product arising in number theory, J. London Math. Soc. 59 (1999), 330-344.

[36] N. S. Larsen, Nonunital semigroup crossed products, Math. Proc. Roy. Irish. Acad. 100A(2) (2000), 205218.

[37] J. Lindiarni, I. Raeburn, Partial-isometric crossed products by semigroups of endomorphisms, J. Operator Theory, 52 (2004), 61-87.

[38] P. S. Muhly, B. Solel, Tensor algebras over $C^{*}$-correspondences (representations, dilations, and $C^{*}-$ envelopes), J. Funct. Anal. 158 (1998), 389-457. 
[39] G. J. Murphy, Crossed products of $C^{*}$-algebras by endomorphisms, Integr. Equ. Oper. Theory 24, (1996), 298-319.

[40] G. J. Murphy, Simplicity of crossed products by endomorphisms, Integr. Equ. Oper. Theory 42 (2002), 90-98.

[41] D. Olesen, G. K. Pedersen, Applications of the Connes spectrum to $C^{*}$-dynamical systems III, J. Funct. Anal. 45 (1982), 357-390.

[42] E. Ortega, E. Pardo, The structure of Stacey cross products by endomorphisms. Operator algebra and dynamics, 239-252, Springer Proc. Math. Stat., 58, Springer, Heidelberg, 2013

[43] W. L. Paschke, The crossed product of a $C^{*}$-algebra by an endomorphism, Proc. Amer. math. Soc. 80 (1980), 113-118.

[44] M. V. Pimsner, A class of $C^{*}$-algebras generalizing both Cuntz-Krieger algebras and crossed products by $\mathbb{Z}$, Fields Institute Communications 12 (1997), 189-212.

[45] M. Rørdam, Classification of certain infinite simple $C^{*}$-algebras, J. Funct. Anal. 131 (1995), 415-458.

[46] J. Schweizer, Dilations of $C^{*}$-correspondences and the simplicity of Cuntz-Pimsner algebras, J. Funct. Anal. 180 (2001), 404-425.

[47] P. J. Stacey, Crossed products of $C^{*}$-algebras by ${ }^{*}$-endomorphisms, J. Austral. Math. Soc. Ser. A 54 (1993), 204-212.

Department of Mathematics and Computer Science, The University of Southern Denmark, Campusvej 55, DK-5230 Odense M, Denmark

Institute of Mathematics, Polish Academy of Science, ul. Śniadeckich 8, PL-00-956 Warszawa, POLAND

Institute of Mathematics, University of Bialystok, ul. Akademicka 2, PL-15-267 Bialystok, POLAND

E-mail address: bartoszk@math.uwb.edu.pl 